TRANSACTIONS OF THE

AMERICAN MATHEMATICAL SOCIETY

Volume 280, Number 1, November 1983

\title{
THE $S^{1}$-TRANSFER IN SURGERY THEORY
}

BY

\author{
H. J. MUNKHOLM AND E. K. PEDERSEN
}

\begin{abstract}
Let $S^{1} \rightarrow X \rightarrow Y$ be an $S^{1}$-bundle of Poincare spaces. If $f: N \rightarrow Y$ is a surgery problem then so is the pullback $\hat{f}: M \rightarrow X$. We define algebraically a homomorphism $\varphi^{!}: L_{n}\left(\mathbf{Z} \pi_{1}(Y)\right) \rightarrow L_{n+1}\left(\mathbf{Z} \pi_{1}(X)\right)$ and prove that it maps the surgery obstruction for $f$ to the one for $\hat{f}$.
\end{abstract}

1. Introduction, statement of results. The purpose of this paper is to describe algebraically the homomorphism of Wall groups induced by pullback to the total space of an $S^{1}$-bundle.

Specifically, let $X \stackrel{p}{\rightarrow} Y$ be an $S^{1}$-bundle of Poincaré spaces, $\operatorname{dim} Y=l$, and let $N \rightarrow Y, \nu_{N} \rightarrow \xi$ be a surgery problem with $Y$ as target. Pullback defines an $S^{1}$-bundle $M \rightarrow N$ and we also get a surgery problem $M \rightarrow X$ (the bundle map also given by pullback).

It is well known, and follows easily from Wall [5, Chapter 9], that this construction defines a homomorphism

$$
p^{*}: L_{l}^{\varepsilon}\left(\mathbf{Z} \rho, w_{Y}\right) \rightarrow L_{l+1}^{\varepsilon}\left(\mathbf{Z} \pi, w_{X}\right), \quad \rho=\pi_{1} Y, \pi=\pi_{1} X,
$$

$\varepsilon=s$ or $h$, which we proceed to describe. To do this we need the following construction: Denote the element of $\pi_{1}(X)$ defined by $S^{1} \subset X$ by $t$ and the bundle orientation map $\rho \rightarrow \mathbf{Z} / 2 \mathbf{Z}$ by $\omega$. In $\mathbf{Z} \pi$ we introduce the following operation:

$$
g^{t}= \begin{cases}g, & \omega(\varphi(g))=1, \\ -g t^{-1}, & \omega(\varphi(g))=-1 .\end{cases}
$$

This operation extends linearly to an automorphism of $\mathbf{Z} \pi$ which in turn induces an automorphism $x \rightarrow x^{t}$ of the ring $M_{r}(\mathbf{Z} \pi)$ of $r \times r$ matrices.

Also let $x \rightarrow x^{*}$ denote the standard anti-involution on $M_{r}(\mathbf{Z} \pi)$; thus, $g^{*}=$ $w_{X}(g) g^{-1}$ for $g \in \pi$. We denote the induced map $\mathbf{Z} \pi \rightarrow \mathbf{Z} \rho$ by $\varphi$.

We may now state our

MAIN THEOREM. The homomorphism

$$
p^{*}: L_{l}^{\varepsilon}\left(\mathbf{Z} \rho ; w_{Y}\right) \rightarrow L_{l+1}^{\varepsilon}\left(\mathbf{Z} \pi ; w_{X}\right)
$$

is given as follows:

Let $l=2 k$ be even and $\alpha \in M_{r}(\mathbf{Z} \rho)$ represent an element (the self-intersection form) $[\alpha] \in L_{2 k}^{\varepsilon}\left(\mathbf{Z} \rho, w_{Y}\right)$. Also choose an arbitrary $\tilde{\alpha} \in M_{r}(\mathbf{Z} \pi)$ with $\varphi(\tilde{\alpha})=\alpha$. Then there

Received by the editors December 8, 1981 and, in revised form, April 28, 1982 and September 14, 1982. 1980 Mathematics Subject Classification. Primary 57R67; Secondary 18F25. 
is a unitary (special unitary if $\varepsilon=s$ ) matrix $\tilde{A} \in M_{2 r}\left(\mathbf{Z}_{\pi}\right)$ of the form

$$
\tilde{A}=\left(\begin{array}{cc}
? & (-1)^{k}\left(t^{-1}-1\right) I_{r} \\
? & \tilde{\alpha}^{t}+(-1)^{k} \tilde{\alpha}^{*} t^{-1}
\end{array}\right)
$$

giving a well-defined element $[\tilde{A}]$ of $L_{2 k+1}^{\varepsilon}\left(\mathbf{Z} \pi, w_{X}\right)$ and $p^{*}([\alpha])=[\tilde{A}]$. Here $I_{r}$ is the $r \times r$ identity matrix. The exact form of the $r \times r$ matrices indicated by ? is immaterial (see [5, Chapter 6]).

Let $l=2 k+1$ and let $A \in M_{r}(\mathbf{Z} \rho)$ be a unitary matrix representing an element of $L_{2 k+1}^{\varepsilon}\left(\mathbf{Z} \rho, w_{Y}\right)$. Choose $\tilde{A} \in M_{2 r}(\mathbf{Z} \pi)$ with $\varphi(\tilde{A})=A$ and write $\tilde{J}$ for the $2 r \times 2 r$ matrix $\left(\begin{array}{ll}0 & 1 \\ 0 & 0\end{array}\right)$. Then there exist $2 r \times 2 r$ matrices $\tilde{\theta}$ and $\tilde{X}$ over $\mathbf{Z} \pi$ so that

$$
\tilde{A}^{*} \tilde{J} \tilde{A}^{t}=t^{-1} \tilde{\theta}^{t}-(-1)^{k} \tilde{\theta}^{*}+\tilde{X}\left(1-t^{-1}\right)+\tilde{J} .
$$

For any such choice, the matrix

$$
W=\left(\begin{array}{cc}
\tilde{K} & \tilde{A} \\
0 & \tilde{X}
\end{array}\right), \quad \text { where } \tilde{K}=\left(\begin{array}{cc}
0 & I_{r} \\
(-1)^{k} t^{-1} I_{r} & 0
\end{array}\right)
$$

represents a well-defined element of $L_{2 k+2}^{\varepsilon}\left(\mathbf{Z} \pi, w_{X}\right)$ (as self-intersection form) and $p^{*}([A])=[W]$.

REMARKS ON DIMENSIONS. There are no dimensional restrictions in the theorem. In low dimensions one defines the surgery obstruction by crossing the surgery problem with $C P^{2}$. Since the geometrically defined transfer is easily seen to commute with crossproduct with $\mathbf{C} P^{2}$, we get that the surgery obstructions are always related as stated in the theorem.

The reader may consult $\$ 2$ for matrix representations of Wall group elements.

The results were announced in the Amer. Math. Soc. Abstracts, February 1981. They were previously known for product bundles [4], and, more generally, for orientable $S^{1}$-bundles where the surjection of fundamental groups is split, [2]. In [3] A. A. Ranicki outlines a program leading to similar results.

2. The algebraic $S^{1}$-transfer map. Let $\varphi: \pi \rightarrow \rho$ be a surjective group homomorphism with cyclic kernel generated by $t \in \pi$. Also let $w_{B}: \rho \rightarrow\{ \pm 1\}, w_{E}: \pi \rightarrow\{ \pm 1\}$ and $\omega: \rho \rightarrow\{ \pm 1\}$ be group homomorphisms related by the identity $w_{E}(g)=$ $\omega(\varphi(g)) w_{B}(g), g \in \pi$. It is the goal of this section to define a homomorphism of Wall groups

$$
\varphi^{!}: L_{l}^{\varepsilon}\left(\rho ; w_{B}\right) \rightarrow L_{l+1}^{\varepsilon}\left(\pi ; w_{E}\right)
$$

called the algebraic $S^{1}$-transfer map $(\varepsilon=s$ or $h)$.

We describe the Wall groups in terms of matrices. This is done briefly in $§ 2 \mathrm{~A}$. In §2B we set up the algebraic situation in which the algebraic $S^{1}$-transfer is naturally defined. Finally in $\$ \S 2 C, D$ we give the definition of $\varphi^{!}$for $l$ even and $l$ odd, respectively.

2A. Wall groups in terms of matrices. Let $R$ be a ring with unit and with a given anti-involution $r \rightarrow r^{*}$. We extend the anti-involution over the ring $M_{m}(R)$ of $m \times m$ matrices over $R$ by putting $\left(a_{i j}\right)^{*}=\left(a_{j i}^{*}\right)$. We shall translate Wall's definition (see [5]) of the groups $L_{l}^{\varepsilon}(R)$ into matrix terms (using also ideas of Ranicki along the way). 
Each element of $L_{2 k}^{\varepsilon}(R)$ is represented by some $\alpha \in M_{r}(R)$ (some $r \geqslant 1$ ) for which

$$
\alpha+(-1)^{k} \alpha^{*} \in G l(r, R) \quad(\text { and is simple, if } \varepsilon=s) .
$$

The sum is defined by

$$
[\alpha]+[\beta]=[\alpha \oplus \beta]
$$

where, as usual, $\alpha \oplus \beta=\left(\begin{array}{cc}\alpha & 0 \\ 0 & \beta\end{array}\right)$. The equivalence relation is generated by

$$
\begin{gathered}
{\left[\left(\begin{array}{ll}
0 & 1 \\
0 & 0
\end{array}\right)\right]=0,} \\
{\left[\alpha+\nu-(-1)^{k} \nu^{*}\right]=[\alpha] \text { for any } \nu \in M_{r}(R),} \\
{\left[\sigma^{*} \alpha \sigma\right]=[\alpha] \quad \text { for any } \sigma \in M_{r}(R) \text { which is invertible }} \\
\text { (and simple if } \varepsilon=s) .
\end{gathered}
$$

Elements of $L_{2 k+1}^{\varepsilon}(R)$ are represented by $2 r \times 2 r$ matrices $A=\left(\begin{array}{c}\alpha \\ \xi \beta\end{array}\right) \in U_{r}^{\varepsilon}(R)$ where $U_{r}^{\varepsilon}=S U_{r}$ if $\varepsilon=s, U_{r}^{\varepsilon}=U_{r}$ if $\varepsilon=h$. This means that

$$
A^{*} J A=J+\theta-(-1)^{k} \theta^{*}
$$

for some $\theta \in M_{2 r}(R)$. Here $J=\left(\begin{array}{ll}0 & 1 \\ 0 & 0\end{array}\right)$ and if $\varepsilon=s$ it is also required that $A$ be simple. Note that $K=J+(-1)^{k} J^{*}$ is invertible and $A^{*} K A=K$ so $A$ is invertible with $A^{-1}=K^{-1} A^{*} K$.

However, from Wall's description of $L_{2 k+1}^{\varepsilon}(R)$ we know that the equivalence class of $A$ depends only on $\left(\begin{array}{c}\eta \\ \beta\end{array}\right)$. Thus we shall describe elements of $L_{2 k+1}^{\varepsilon}(R)$ as equivalence classes $\left[\begin{array}{l}\eta \\ \beta\end{array}\right]$ of such $2 r \times r$ matrices $\left(\begin{array}{l}\eta \\ \beta\end{array}\right)$. The representatives must satisfy

$$
\begin{gathered}
\eta^{*} \beta=\nu-(-1)^{k} \nu^{*} \quad \text { for some } \nu \in M_{r}(R), \\
\left(\begin{array}{l}
\eta \\
\beta
\end{array}\right): R^{r} \rightarrow R^{2 r} \quad \text { is a split injection. }
\end{gathered}
$$

The sum is given by

$$
\left[\begin{array}{l}
\eta_{1} \\
\beta_{1}
\end{array}\right]+\left[\begin{array}{l}
\eta_{2} \\
\beta_{2}
\end{array}\right]=\left[\begin{array}{l}
\eta_{1} \oplus \eta_{2} \\
\beta_{1} \oplus \beta_{2}
\end{array}\right] .
$$

Finally, the equivalence relation is generated by

$$
\begin{gathered}
{\left[\begin{array}{l}
1 \\
0
\end{array}\right]=0,} \\
{\left[\begin{array}{l}
\eta \\
\beta
\end{array}\right]=\left[\begin{array}{c}
\beta \\
(-1)^{k} \eta
\end{array}\right],} \\
{\left[\begin{array}{l}
\eta \\
\beta
\end{array}\right]=\left[\begin{array}{c}
\left(\sigma^{*}\right)^{-1} \eta \\
\sigma \beta
\end{array}\right]=\left[\begin{array}{c}
\eta \sigma \\
\beta \sigma
\end{array}\right]}
\end{gathered}
$$

for any $\sigma \in M_{r}(R)$ which is invertible (and simple if $\varepsilon=s$ ),

$$
\left[\begin{array}{l}
\eta \\
\beta
\end{array}\right]=\left[\begin{array}{c}
\eta \\
\left(\nu-(-1)^{k} \nu^{*}\right) \eta+\beta
\end{array}\right] .
$$


To see that, indeed, these relations do hold, note that in terms of the full unitary matrix $A=\left(\begin{array}{c}\alpha \\ \xi \beta\end{array}\right)$ they come from left (or right in case of the second part of (2A.12)) multiplication by

$$
\left(\begin{array}{cc}
0 & 1 \\
(-1)^{k} & 0
\end{array}\right), \quad\left(\begin{array}{cc}
\left(\sigma^{*}\right)^{-1} & 0 \\
0 & \sigma
\end{array}\right) \quad \text { and }\left(\begin{array}{cc}
1 & 0 \\
\nu-(-1)^{k} \nu^{*} & 1
\end{array}\right)
$$

respectively. To see that the relations suffice to describe $L_{2 k+1}^{\varepsilon}(R)$, note that they include left multiplication by a full set of generators of $T U^{\varepsilon}(R)$ (compare $\S 6$ of Wall [5]).

2B. The algebraic set up for $\varphi !$. Let $R$ be a ring with unit. Suppose given

an anti-involution $r \rightarrow r^{*}$ of $R$,

an automorphism $r \rightarrow r^{t}$ of $R$, and

a unit $t \in R$

such that the following identities hold:

$$
\begin{gathered}
r^{* t}=r^{* *}, \\
(t-1) r=r^{t}(t-1), \\
t r t^{-1}=r^{t^{2}} \quad\left(=\left(r^{t}\right)^{t}\right), \\
t^{*}=t^{-1}, \\
t^{t}=t .
\end{gathered}
$$

Then the ideal $(t-1) R$ is 2 -sided so we have the projection $\varphi: R \rightarrow \bar{R}=R /(t-1) R$. By (2B.4-5), $\bar{R}$ inherits an anti-involution $\bar{*}$ and an automorphism $\bar{t}\left(\bar{r} \rightarrow \bar{r}^{\bar{t}}=\varphi\left(r^{t}\right)\right.$, if $\bar{r}=\varphi(r)$ ). By (2B.3), $\bar{t}$ is an involution. To make notation fit with the standard one in the prime example (see (2B.16)) we shall write $*$ for the composite anti-involution $\bar{*} \bar{t}=\bar{t} \bar{*}$ on $\bar{R}$. Thus we have anti-involutions in $R$ and $\bar{R}$ which are both called $*$. But they do not correspond under $\varphi$. Instead, one has

$$
\varphi(r)^{*}=\varphi\left(r^{* *}\right) .
$$

We shall have occasion to also use $t^{-1} \in R$ and the inverse automorphism $r \rightarrow r^{t^{-1}}$. To shorten notation let us write $s$ for $t^{-1}$ in both instances. Note that we then have

$$
\begin{gathered}
r^{* s}=r^{s *}, \\
(s-1) r=r^{s}(s-1), \\
s r t=r^{s^{2}}, \\
s^{*}=t, \\
s^{s}=s, \\
\varphi(r)^{*}=\varphi\left(r^{s *}\right), \\
s^{t}=s, \quad t^{s}=t, \\
s t=t s=1 \quad \text { (in two senses). }
\end{gathered}
$$

We extend the anti-involutions to the matrix rings $M_{r}(R)$ and $M_{r}(\bar{R})$ by using them on each entry and transposing. And we extend the automorphisms $s, t$ to $M_{r}(R)$ by using them on each entry. We can identify $M_{r}(R) /(t-1) M_{r}(R)$ with $M_{r}(\bar{R})$ in the 
obvious way. The projection $M_{r}(R) \rightarrow M_{r}(\bar{R})$ then is the map of matrix rings induced by $\varphi$. We continue to call it $\varphi$. All the above identities then make sense, and are true, when we let $r \in M_{r}(R)$.

(2B.15) EXAMPLE. By a set of fundamental groups and orientation data we shall understand an exact sequence of groups

$$
\mathbf{Z} \stackrel{i}{\rightarrow} \pi \stackrel{\varphi}{\rightarrow} \rho \rightarrow\{1\}
$$

with a compatible action $\omega: \rho \rightarrow\{ \pm 1\}$ (i.e. $\left.i(\omega(\varphi(g))(n))=g i(n) g^{-1}, n \in \mathbf{Z}, g \in \pi\right)$ and with orientation homomorphisms $w_{B}: \rho \rightarrow\{ \pm 1\}, w_{E}: \pi \rightarrow\{ \pm 1\}$, satisfying

$$
w_{E}(g)=w_{B}(\varphi(g)) \omega(\varphi(g)), \quad g \in \pi .
$$

Let there be given such a set and a commutative ring $A$ with unit. We take $R=A \pi$, the group ring. We let $t=i(1)$ and we define $*$ and $t$ on a group element $g \in \pi$ by

$$
\begin{aligned}
g^{*}=w_{E}(g) g^{-1}, \quad g^{t} & =g \quad \text { if } \omega(\varphi(g))=1, \\
& =-g s \quad \text { if } \omega(\varphi(g))=-1 .
\end{aligned}
$$

The conditions (2B.1-5) are easily verified. Also, one can identify the projection $R \rightarrow \bar{R}$ with $\varphi: A \pi \rightarrow A \rho$ (induced by $\varphi: \pi \rightarrow \rho$ ). Then the induced anti-involution on $A \rho$ becomes the standard one, i.e.

$$
\bar{g}^{*}=w_{B}(\bar{g}) \bar{g}^{-1}, \quad \bar{g}^{\bar{t}}=\omega(\bar{g}) \bar{g}, \quad \bar{g} \in \rho .
$$

(2B.17) EXAMPLE. The above algebraic example comes from geometry. In fact, if $f$ : $E \rightarrow B$ is a locally trivial manifold bundle with fibre $S^{1}$ then the homotopy sequence $\pi_{1}\left(S^{1}\right) \rightarrow \pi_{1}(E) \rightarrow \pi_{1}(B) \rightarrow 1$, together with the orientation maps $w_{B}, w_{E}$ and the action of $\pi_{1}(B)$ on $\pi_{1}\left(S^{1}\right)$, fits into the scheme.

2C. Definition of $\varphi^{!}: L_{2 k}^{\varepsilon}(\bar{R}) \rightarrow L_{2 k+1}^{\varepsilon}(R)$. Let $\varphi: R \rightarrow \bar{R}$ be as in $\$ 2 \mathrm{~B}$.

Abuse of language. In defining $L_{l}^{s}(\bar{R})$ we allow torsion in $\{ \pm 1\} \subseteq K_{1}(\bar{R})$, but in $L_{l}^{s}(R)$ we allow torsion in $\left\{ \pm t^{i} \mid i \in \mathbf{Z}\right\} \subseteq K_{1}(R)$.

Of course, if $R=\mathbf{Z} \pi, \bar{R}=\mathbf{Z} \rho$ as in Example 2B.15, we may allow torsion in $\{ \pm \pi\}$, respectively $\{ \pm \rho\}$, when we define $L_{l}^{s}$, to give the usual definition of $L_{l}^{s}$ (group ring). With both interpretations we have

(2C.1) THEOREM. If $\alpha \in M_{r}(\bar{R})$ represents an element $[\alpha] \in L_{2 k}^{\varepsilon}(\bar{R})$ and $\tilde{\alpha} \in M_{r}(R)$ has $\varphi(\tilde{\alpha})=\alpha$, then

$$
\left(\begin{array}{l}
\eta \\
\beta
\end{array}\right)=\left(\begin{array}{c}
(-1)^{k}(s-1) \\
\tilde{\alpha}^{t}+(-1)^{k} \tilde{\alpha}^{*} s
\end{array}\right)
$$

represents an element of $L_{2 k+1}^{\varepsilon}(R)$ and

$$
\varphi^{!}([\alpha])=\left[\begin{array}{l}
\eta \\
\beta
\end{array}\right]
$$

defines a homomorphism $\varphi^{!}: L_{2 k}^{\varepsilon}(\bar{R}) \rightarrow L_{2 k+1}^{\varepsilon}(R)$.

Proof. We first verify (2A.7-8). Since $\varphi(\beta)=\left(\alpha+(-1)^{k} \alpha^{*}\right)^{\bar{t}}$ is known to be an isomorphism, there must exist $\gamma, \delta \in M_{r}(R)$ with $\gamma \beta+\delta(s-1)=1$, i.e. 
$\left((-1)^{k} \delta, \gamma\right)\left(\begin{array}{l}\eta \\ \beta\end{array}\right)=1$. Also,

$$
\eta^{*} \beta=\nu-(-1)^{k} \nu^{*} \quad \text { where } \nu=(-1)^{k}(t-1) \tilde{\alpha}^{t} .
$$

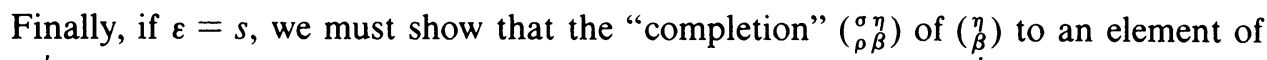
$U_{r}^{h}(R)$ (see (2A.6)) actually is simple. From the definition of $U_{r}^{h}(R)$ we know that $\sigma^{*} \beta+(-1)^{k} \rho^{*} \eta=1$. Now, apply $(\cdot)^{s *}$ and note that $\beta^{s *}=(-1)^{k} \beta t$. You then get

$$
\beta(-1)^{k} t \sigma^{s}=1-\rho(t-1) \text {. }
$$

Since $\beta$ lifts the simple matrix $\alpha+(-1)^{k} \alpha^{*}$, it follows from Theorem 1.1 of Munkholm and Pedersen [1] that the matrix

$$
\left(\begin{array}{cc}
\beta & -\rho \\
t-1 & (-1)^{k} t \sigma
\end{array}\right)
$$

is simple. Hence, so is $\left(\begin{array}{c}\sigma \eta \\ \rho \beta\end{array}\right)$, which equals

$$
\left(\begin{array}{cc}
0 & (-1)^{k+1} s \\
1 & 0
\end{array}\right)\left(\begin{array}{cc}
\beta & -\rho \\
t-1 & (-1)^{k} t \sigma
\end{array}\right)\left(\begin{array}{cc}
0 & 1 \\
-1 & 0
\end{array}\right) .
$$

We go on to show that $\left[\begin{array}{l}\eta \\ \beta\end{array}\right]$ depends at most on $\alpha$ (i.e. not on the lifting $\tilde{\alpha}$ ). In fact, any other lifting has the form $\tilde{\alpha}_{1}=\tilde{\alpha}+(s-1) \tilde{\nu}$ and this makes

$$
\beta_{1}=\beta+\left(\tilde{\nu}-(-1)^{k} \tilde{\nu}^{*}\right)(s-1),
$$

so a reference to (2A.13) settles the matter.

We next note that $\varphi^{!}\left(\alpha_{1} \oplus \alpha_{2}\right)=\varphi^{!}\left(\alpha_{1}\right)+\varphi^{!}\left(\alpha_{2}\right)$. Thus, we just have to show that the relations (2A.3-5) are respected. If $\alpha=\left(\begin{array}{ll}0 & 1 \\ 0 & 0\end{array}\right)$ then $\left[\begin{array}{l}\eta \\ \beta\end{array}\right]$ becomes

$$
\left[\begin{array}{cc}
(-1)^{k}(s-1) & 0 \\
0 & (-1)^{k}(s-1) \\
0 & 1 \\
(-1)^{k} s & 0
\end{array}\right]=\left[\begin{array}{cc}
0 & 1 \\
(-1)^{k} s & 0 \\
0 & 0 \\
0 & 0
\end{array}\right]=\left[\begin{array}{ll}
1 & 0 \\
0 & 1 \\
0 & 0 \\
0 & 0
\end{array}\right]=0+0=0 .
$$

Here, for the first equality we have used (2A.11) followed by (2A.13) with $\nu=\left(\begin{array}{ll}0 & 0 \\ 1-s & 0\end{array}\right)$. Next (2A.12) with the obvious $\sigma$ and finally (2A.9-10).

If we replace $\alpha$ by $\alpha+\nu-(-1)^{k} \nu^{*}$ then we may keep $\beta$ unchanged by replacing $\tilde{\alpha}$ by $\tilde{\alpha}+\tilde{\nu}-(-1)^{k}(\tilde{\nu} t)^{t *}$. Thus (2A.4) is respected.

Finally we consider (2A.5). If we replace $\alpha$ by $\sigma^{*} \alpha \sigma$ where $\sigma$ is simple, then we can lift $\sigma$ to a simple isomorphism $\tilde{\sigma}$. We can replace $\tilde{\alpha}$ by $\tilde{\sigma}^{s *} \tilde{\alpha} \tilde{\sigma}$. Then $\beta$ is replaced by $\tilde{\sigma}^{*} \beta \tilde{\sigma}^{t}$. Since $\tilde{\sigma}^{-1} \eta \tilde{\sigma}^{t}=\eta$ we see from (2A.12) that $\left[\begin{array}{l}\eta \\ \beta\end{array}\right]$ is unaffected. When $\sigma$ is not simple we first stabilize, i.e. replace $\alpha$ by $\alpha+J$ (recall $\left.J=\left(\begin{array}{ll}0 & 1 \\ 0 & 0\end{array}\right)\right)$. Since $\sigma \oplus \tau \oplus 1$ (where $\tau=\sigma^{-1}$ ) is simple we already know that $\alpha$ and the matrix

$$
(\sigma \oplus \tau \oplus 1)^{*}(\alpha \oplus J)(\sigma+\tau \oplus 1)=\sigma^{*} \alpha \sigma \oplus\left(\begin{array}{cc}
0 & \tau^{*} \\
0 & 0
\end{array}\right)
$$


give rise to the same element of $L_{2 k+1}^{h}(R)$. Thus, it suffices to show that $\left(\begin{array}{ll}0 & \tau^{*} \\ 0 & 0\end{array}\right)$ gives rise to the trivial element. Write $\tilde{\tau}$ for some matrix with $\varphi(\tilde{\tau})=\tau^{\bar{t}}$. We have to show that

$$
\left(\begin{array}{cc}
(-1)^{k}(s-1) & 0 \\
0 & (-1)^{k}(s-1) \\
0 & \tilde{\tau}^{*} \\
(-1)^{k} \tilde{\tau}^{s} s & 0
\end{array}\right)
$$

represents 0 . We add some copies of $\left(\begin{array}{l}1 \\ 0\end{array}\right)$ and use (2A.12) to permute columns and rows until we get to

$$
\left(\begin{array}{cccc}
(-1)^{k}(s-1) & 0 & 0 & 0 \\
0 & 1 & 0 & 0 \\
0 & 0 & (-1)^{k}(s-1) & 0 \\
0 & 0 & 0 & 1 \\
0 & 0 & \tilde{\tau}^{*} & 0 \\
0 & 0 & 0 & 0 \\
(-1)^{k} \tilde{\tau}^{s} s & 0 & 0 & 0 \\
0 & 0 & 0 & 0
\end{array}\right) .
$$

From Theorem 1.1 of [1] we know that there exists an invertible matrix of the form

$$
A=\left(\begin{array}{lc}
\tilde{\tau}^{s *} & -\tilde{\gamma} \\
t-1 & \tilde{\rho}^{t}
\end{array}\right)
$$

(it suffices to choose $\tilde{\gamma}$ and $\tilde{\rho}$ so that $\tilde{\tau}^{s *} \tilde{\rho}=1-\tilde{\gamma}(t-1)$ ). Now apply (2A.13) with

$$
\nu=\left(\begin{array}{cccc}
0 & 0 & 0 & -\tilde{\gamma}^{t} \\
0 & 0 & (-1)^{k+1} t & t \tilde{\rho} s \\
0 & 0 & 0 & 0 \\
0 & 0 & 0 & 0
\end{array}\right)
$$

This brings you to the matrix

$$
\left(\begin{array}{cc}
X & 0 \\
0 & X \\
0 & A^{t} \\
B & 0
\end{array}\right) \quad \text { where } X=\left(\begin{array}{cc}
(-1)^{k}(s-1) & 0 \\
0 & 1
\end{array}\right), B=\left(\begin{array}{cc}
(-1)^{k} \tilde{\tau}^{s} & s \\
\tilde{\gamma}^{t *}(s-1) & (-1)^{k+1} t \tilde{\rho}^{*} s
\end{array}\right)
$$

and $A$ is as above. Now $B$ is easily seen to be invertible. Thus the matrix we arrived at is equivalent to (use (2A.12))

$$
\left(\begin{array}{cc}
0 & X B^{-1} \\
X\left(A^{t}\right)^{-1} & 0 \\
1 & 0 \\
0 & 1
\end{array}\right)
$$

which represents zero provided $X B^{-1}=(-1)^{k+1}\left(X\left(A^{t}\right)^{-1}\right)^{*}$ (use (2A.11) and (2A.13)). And the latter identity is easily checked. 
2D. Definition of $\varphi^{!}: L_{2 k+1}^{\varepsilon}(\bar{R}) \rightarrow L_{2 k+2}^{\varepsilon}(R)$.

Abuse of language (as in $\S 2 \mathrm{C})$. Recall that $J=\left(\begin{array}{ll}0 & 1 \\ 0 & 0\end{array}\right) \in M_{2 r}(\bar{R}), K=J+(-1)^{k} J^{*}$. Let $\tilde{J}=\left(\begin{array}{ll}0 & 1 \\ 0 & 0\end{array}\right) \in M_{r}(R)$,

$$
\tilde{K}=\tilde{J}+(-1)^{k} \tilde{J}^{*} s=\left(\begin{array}{cc}
0 & 1 \\
(-1)^{k} s & 0
\end{array}\right)
$$

and note that $\tilde{K}^{*}=\tilde{K}^{-1}$.

If $[A] \in L_{2 k+1}^{\varepsilon}(\bar{R})$ and if $\tilde{A} \in M_{2 r}(R)$ has $\varphi(\tilde{A})=A$, then we see from (2A.6) that there exist $2 r \times 2 r$ matrices $\tilde{\theta}$ and $\tilde{X}$ over $R$ so that

$$
\tilde{A}^{s *} \tilde{J} \tilde{A}=s \tilde{\theta}-(-1)^{k} \tilde{\theta}^{s *}+(1-s) \tilde{X}+\tilde{J} .
$$

Since $s=t^{-1}$ this is the relation mentioned in the Main Theorem of the introduction.

(2D.2) ThEOREM. For any choice of $\tilde{A}, \tilde{\theta}$ and $\tilde{X}$ as in (2D.1), the matrix

$$
W=\left(\begin{array}{cc}
\tilde{K} & \tilde{A} \\
0 & \tilde{X}
\end{array}\right)
$$

represents an element of $L_{2 k+2}^{\varepsilon}(R)$, and $\varphi^{\prime}([A])=[W]$ defines a homomorphism $\varphi^{\prime}$ : $L_{2 k+1}^{\varepsilon}(\bar{R}) \rightarrow L_{2 k+2}^{\varepsilon}(R)$.

Proof. We first show that $W$ really represents an element of $L_{2 k+2}^{\varepsilon}(R)$, i.e. that $\Lambda=W+(-1)^{k+1} W^{*}$ is a (simple if $\varepsilon=s$ ) isomorphism. Applying $(-1)^{k}(\cdot)^{s *} s$ to (2D.1), and adding the results to (2D.1) one gets

$$
\tilde{A}^{s *} \tilde{K} \tilde{A}=\tilde{K}+(1-s)\left(\tilde{X}+(-1)^{k+1} \tilde{X}^{*}\right)
$$

from which

$$
\tilde{A}^{s *} \tilde{K} \tilde{A} \tilde{K}^{*}=1+(t-1) s\left(\tilde{X}+(-1)^{k+1} \tilde{X}^{*}\right) \tilde{K}^{*} .
$$

From Theorem 1.1 of Munkholm and Pedersen [1] it then follows that

$$
\left(\begin{array}{cc}
\tilde{A}^{s *} \tilde{K} & s\left(\tilde{X}+(-1)^{k+1} \tilde{X}^{*}\right)^{t} \tilde{K}^{*} \\
t-1 & \tilde{A}^{t} \tilde{K}^{*}
\end{array}\right)
$$

is a (simple, if $\varepsilon=s$ ) isomorphism. Now apply $t$, multiply on the right by

$$
\left(\begin{array}{cc}
(-1)^{k+1} \tilde{K}^{*} & 0 \\
0 & \tilde{K} t
\end{array}\right)
$$

and on the left by $\left(\begin{array}{l}0 s \\ 1\end{array}\right)$. The result is a (simple, if $\left.\varepsilon=s\right)$ isomorphism which happens to be $\Lambda$.

We proceed to show that, for a fixed $A,[W]$ is independent of the choice of $\tilde{A}, \tilde{\theta}$ and $\tilde{X}$. First note that the kernel of $\varphi: M_{2 r}(R) \rightarrow M_{2 r}(\bar{R})$ is generated hy

$$
\tilde{L}=\tilde{K}+(-1)^{k+1} \tilde{K}^{*}=\left(\begin{array}{cc}
0 & 1-t \\
(-1)^{k}(s-1) & 0
\end{array}\right) .
$$

Thus any new choice of $\tilde{A}$ must have the form $\tilde{A}_{1}=\tilde{A}+\tilde{L} \tilde{B}$ for some $\tilde{B} \in M_{2 r}(R)$. In (2A.6) one may compensate by taking $\tilde{\theta}_{1}=\tilde{\theta}$ and

$$
\tilde{X}_{1}=\tilde{X}+\tilde{B}^{*} \tilde{J}^{*} \tilde{J} \tilde{A}-(-1)^{k} \tilde{A}^{*} \tilde{J} \tilde{J}^{*} \tilde{B}+(-1)^{k+1} \tilde{B}^{*}(1-s) \tilde{J}^{*} \tilde{B}
$$


(for this computation use $\tilde{J} \tilde{L}=(-1)^{k}(s-1) \tilde{J} \tilde{J}^{*}, \tilde{L}^{s *} \tilde{J}=(1-s) \tilde{J}^{*} \tilde{J}$ and $\tilde{J}^{*} \tilde{J} \tilde{J}^{*}=$ $\left.\tilde{J}^{*}\right)$. A simple computation then shows that

$$
\left(\begin{array}{cc}
1 & 0 \\
\tilde{B}^{*} & 1
\end{array}\right)\left(\begin{array}{cc}
\tilde{K} & \tilde{A} \\
0 & \tilde{X}
\end{array}\right)\left(\begin{array}{cc}
1 & \tilde{B} \\
0 & 1
\end{array}\right)-\left(\begin{array}{cc}
\tilde{K} & \tilde{A}_{1} \\
0 & \tilde{X}_{1}
\end{array}\right)
$$

has the form $\nu-(-1)^{k+1} \nu^{*}$ with

$$
\nu=\left(\begin{array}{cc}
0 & 0 \\
\tilde{B}^{*} \tilde{K} & \tilde{B}^{*} \tilde{J} \tilde{B}+\tilde{B}^{*} \tilde{J} \tilde{J}^{*} \tilde{A}
\end{array}\right)
$$

(note that $\tilde{J}^{*}+\tilde{J}^{*} \tilde{J}=1$ ).

This shows that any change in the lifting $\tilde{A}$ can be followed by a change in $\tilde{X}$ so that $[W]$ is unaltered. Thus, we have to show that, for some choice of $\tilde{A},[W]$ is independent of the choice of $\tilde{\theta}$ and $\tilde{X}$. We first need the following: If you stabilize $A$ then you may stabilize $\tilde{A}, \tilde{\theta}$ and $\tilde{X}$ in such a manner that $[W]$ is unchanged. We leave the straightforward proof to the reader after remarking that when we write the matrices in question out as $2 \times 2$ matrices consisting of $r \times r$ matrices then the stabilizations take the form

$$
\begin{aligned}
& \tilde{A}=\left(\begin{array}{ll}
\tilde{\alpha} & \tilde{\eta} \\
\tilde{\xi} & \tilde{\beta}
\end{array}\right) \rightarrow\left(\begin{array}{ll}
\tilde{\alpha} \oplus 1 & \tilde{\eta} \oplus 0 \\
\tilde{\xi} \oplus 0 & \tilde{\beta} \oplus 1
\end{array}\right), \\
& \tilde{\boldsymbol{\theta}}=\left(\begin{array}{ll}
\tilde{\theta}_{11} & \tilde{\theta}_{12} \\
\tilde{\theta}_{21} & \tilde{\theta}_{22}
\end{array}\right) \rightarrow\left(\begin{array}{ll}
\tilde{\theta}_{11} \oplus 0 & \tilde{\theta}_{12} \oplus 0 \\
\tilde{\theta}_{21} \oplus 0 & \tilde{\theta}_{22} \oplus 0
\end{array}\right), \\
& \tilde{X}=\left(\begin{array}{ll}
\tilde{x}_{11} & \tilde{x}_{12} \\
\tilde{x}_{21} & \tilde{x}_{22}
\end{array}\right) \rightarrow\left(\begin{array}{ll}
\tilde{x}_{11} \oplus 0 & \tilde{x}_{12} \oplus 0 \\
\tilde{x}_{21} \oplus 0 & \tilde{x}_{22} \oplus 0
\end{array}\right) .
\end{aligned}
$$

(2D.6) Lemma. After a possible stabilization of $A$, one can choose a lifting $\tilde{A}$ for which there exist matrices $\tilde{a}, \tilde{z}, \tilde{y}, \tilde{b} \in M_{r}(R)$ such that

$$
\left(\begin{array}{cc}
\tilde{a} & \tilde{\eta} \\
\tilde{z} & \tilde{\beta}
\end{array}\right) \text { and }\left(\begin{array}{cc}
\tilde{\alpha} & \tilde{y} \\
\tilde{\xi} & \tilde{b}
\end{array}\right)
$$

are simple isomorphisms. For any such choice the quadratic form $W$ of Theorem 2D.2 is equivalent to

$$
W_{2}=\left(\begin{array}{cc}
(-1)^{k} \tilde{z}^{s *}(s-1) \tilde{a}^{s} & \tilde{z}^{s *} s \tilde{\eta}+(-1)^{k} \tilde{a}^{s *} \tilde{\beta} \\
0 & \tilde{x}_{22}
\end{array}\right)
$$

and to

$$
W_{2}^{\prime}=\left(\begin{array}{cc}
(-1)^{k} \tilde{b}^{s *}(s-1) \tilde{y}^{s} & (-1)^{k} \tilde{b}^{s *} s \tilde{\alpha}+\tilde{\eta}^{s * \tilde{\xi}} \\
0 & \tilde{x}_{11}
\end{array}\right)
$$

Corollary. $[W]$ is independent of the choice of $\tilde{\theta}, \tilde{X}$.

Proof of Corollary. The equation (2D.1) is equivalent to the following four identities:

$$
\tilde{\boldsymbol{\alpha}}^{s * \tilde{\xi}}=s \tilde{\theta}_{11}-(-1)^{k} \tilde{\theta}_{11}^{s *}+(1-s) \tilde{x}_{11}
$$




$$
\begin{gathered}
\tilde{\alpha}^{s *} \tilde{\beta}=s \tilde{\theta}_{12}-(-1)^{k} \tilde{\theta}_{21}^{s *}+(1-s) \tilde{x}_{12}+1, \\
\tilde{\eta}^{s *} \tilde{\xi}=s \tilde{\theta}_{21}-(-1)^{k} \tilde{\theta}_{12}^{s *}+(1-s) \tilde{x}_{21}, \\
\tilde{\eta}^{s *} \tilde{\beta}=s \tilde{\theta}_{22}-(-1)^{k} \tilde{\theta}_{22}^{s *}+(1-s) \tilde{x}_{22} .
\end{gathered}
$$

Here $\tilde{\theta}_{22}, \tilde{x}_{22}$ enters only in (2D.10) and $\tilde{\theta}_{11}, \tilde{x}_{11}$ only in (2D.7). Therefore, one can obtain any variation in $\tilde{\theta}, \tilde{X}$ by first varying $\tilde{\theta}_{11}, \tilde{\theta}_{12}, \tilde{\theta}_{21}, \tilde{x}_{11}, \tilde{x}_{12}, \tilde{x}_{21}$ while keeping $\tilde{\theta}_{22}, \tilde{x}_{22}$ fixed and next interchanging the roles of the indices 1 and 2 . The fact that $W_{2}$ depends only on $\tilde{a}, \tilde{z}, \tilde{\eta}, \tilde{\beta}$ and $\tilde{x}_{22}$ shows that the first variation leaves $[W]$ fixed. Similarly, one uses $W_{2}^{\prime}$ to handle the second variation.

Proof of Lemma 2D.6. After stabilization we can assume that the Whitehead torsion of $A$ is represented by an $r \times r$ matrix, say $b^{-1}$. Then $A(b \oplus 1)$ and $A(1 \oplus b)$ are simple so they can be lifted to simple matrices over $R$, at least after stabilization. We can now take

$$
\left(\begin{array}{cc}
\tilde{a} & \tilde{\eta} \\
\tilde{z} & \tilde{\beta}
\end{array}\right) \text { and }\left(\begin{array}{cc}
\tilde{\alpha} & \tilde{y} \\
\tilde{\xi} & \tilde{b}
\end{array}\right)
$$

to be liftings of $A(b \oplus 1)$ and $A(1 \oplus b)$, respectively. Let

$$
\Phi=\left(\begin{array}{cccc}
t \tilde{z}^{s} & 0 & 0 & t \tilde{\beta}^{s} \\
(-1)^{k} \tilde{a}^{s} & 0 & 0 & (-1)^{k} \tilde{\eta}^{s} \\
0 & 0 & 1 & 0 \\
0 & 1 & 0 & (-1)^{k}(t-1)
\end{array}\right)
$$

and

$$
\nu=\left(\begin{array}{cccc}
(-1)^{k} \tilde{z}^{s *} \tilde{a}^{s} & 0 & 0 & \tilde{a}^{s *} t \tilde{\beta}^{s}+(-1)^{k} \tilde{z}^{s *} \tilde{\eta}^{s} \\
0 & 0 & 0 & (-1)^{k} \tilde{x}_{22}(t-1) \\
0 & \tilde{x}_{12} & 0 & (-1)^{k} \tilde{x}_{12}(t-1) \\
0 & 0 & 0 & (1-s) \tilde{\theta}_{22}^{s}+(-1)^{k} \tilde{\beta}^{s *} \tilde{\eta}^{s}
\end{array}\right)
$$

Then $\Phi$ is a simple isomorphism and the identities (2D.7-10), together with those derived from them by applying the operation $(\cdot)^{s *} s$, suffice to show that

$$
\Phi^{*} W \Phi=\left(\begin{array}{cccc}
\left(W_{2}\right) & & \tilde{u} & 0 \\
& & \tilde{v} & 0 \\
0 & 0 & \tilde{x}_{11} & 0 \\
0 & 0 & s & 0
\end{array}\right)+\nu-(-1)^{k+1} \nu^{*}
$$

where $\tilde{u}=\tilde{z}^{s *} s \tilde{\alpha}+(-1)^{k} \tilde{a}^{s *} \tilde{\xi}, \tilde{v}=\tilde{x}_{21}-(-1)^{k} \tilde{x}_{12}^{*}$. Finally, conjugation by

$$
\left(\begin{array}{cccc}
1 & 0 & 0 & 0 \\
0 & 1 & 0 & 0 \\
0 & 0 & 1 & 0 \\
-s \tilde{u}^{*} & -s \tilde{v}^{*} & -s \tilde{x}_{11} & t
\end{array}\right)
$$

takes the right-hand matrix into a stabilization of $W_{2}$. 
The proof that $W$ is equivalent to $W_{2}^{\prime}$ is similar. It uses

$$
\begin{aligned}
& \Phi^{\prime}=\left(\begin{array}{cccc}
(-1)^{k} t \tilde{b}^{s} & 0 & 0 & t \tilde{\xi}^{s} \\
\tilde{y}^{s} & 0 & 0 & (-1)^{k} \tilde{\alpha}^{s} \\
0 & 1 & 0 & (-1)^{k}(t-1) \\
0 & 0 & (-1)^{k} & 0
\end{array}\right), \\
& \boldsymbol{\nu}^{\prime}=\left(\begin{array}{cccc}
\tilde{y}^{s *} \tilde{b}^{s} & 0 & 0 & (-1)^{k} \tilde{y}^{s *} t \tilde{\xi}^{s}+\tilde{b}^{s *} \tilde{\alpha}^{s} \\
0 & 0 & \tilde{x}_{21}^{*} & (-1)^{k} \tilde{x}_{11}(t-1) \\
0 & 0 & 0 & \tilde{x}_{21}(t-1) \\
0 & 0 & 0 & (1-s) \tilde{\theta}_{11}^{s}+\tilde{\alpha}^{s *} \tilde{\xi}^{s}
\end{array}\right)
\end{aligned}
$$

and otherwise proceeds as above.

The following description of $[W]$ as a cokernel is inspired by A. A. Ranicki [3]. We do not use it anywhere in the following, but it does fit nicely with the geometric considerations in $\$ 4 \mathrm{~F}$.

(2D.11) Proposition. Let $\tilde{a}, \tilde{z}, \tilde{\eta}, \tilde{\beta}, \tilde{\theta}_{22}, \tilde{x}_{22}$ be as above. Let

$$
W_{3}=\left(\begin{array}{ccc}
0 & 1-t & \tilde{\eta} \\
0 & 0 & \tilde{\beta} \\
0 & 0 & \tilde{x}_{22}
\end{array}\right)
$$

The radical of the quadratic form $\left(R^{3 r}, W_{3}\right)$ is the image of

$$
\left(\begin{array}{c}
t \tilde{\beta}^{s} \\
(-1)^{k} \tilde{\eta}^{s} \\
(-1)^{k}(t-1)
\end{array}\right): R^{r} \rightarrow R^{3 r}
$$

and the cokernel of this map, with its induced quadratic form, represents $[W]$.

Proof. Direct computations show that

$$
\left(W_{3}+(-1)^{k+1} W_{3}^{*}\right)\left(\begin{array}{c}
t \tilde{\beta}^{s} \\
(-1)^{k} \tilde{\eta}^{s} \\
(-1)^{k}(t-1)
\end{array}\right)=\left(\begin{array}{l}
0 \\
0 \\
0
\end{array}\right)
$$

and

$$
\left(\tilde{\beta}^{s *} s,(-1)^{k} \tilde{\eta}^{s *},(-1)^{k}(s-1)\right) W_{3}\left(\begin{array}{c}
t^{s} \\
(-1)^{k} \tilde{\eta}^{s} \\
(-1)^{k}(t-1)
\end{array}\right)=\nu-(-1)^{k+1} \nu^{*}
$$


where $\nu=(1-s) \tilde{\theta}_{22}^{s}$. (These computations really form a subset of those in the proof of Lemma 2D.6). Hence the radical contains the image claimed. Since

$$
\left(\begin{array}{ccc}
t \tilde{z}^{s} & 0 & t \tilde{\beta}^{s} \\
(-1)^{k} \tilde{a}^{s} & 0 & (-1)^{k} \tilde{\eta}^{s} \\
0 & 1 & (-1)^{k}(t-1)
\end{array}\right)
$$

is invertible (and simple if $\varepsilon=s$ ),

$$
\left(\begin{array}{cc}
t \tilde{z}^{s} & 0 \\
(-1)^{k} \tilde{a}^{s} & 0 \\
0 & 1
\end{array}\right)
$$

splits the cokernel involved. Since

$$
\left(\begin{array}{cc}
t \tilde{z}^{s} & 0 \\
(-1)^{k} \tilde{a}^{s} & 0 \\
0 & 1
\end{array}\right)^{*} W_{3}\left(\begin{array}{cc}
t \tilde{z}^{s} & 0 \\
(-1)^{k} \tilde{a}^{s} & 0 \\
0 & 1
\end{array}\right)=W_{2},
$$

the proof is complete.

We now know that $[W]$ depends only on $A$. We must check that it depends only on $[A] \in L_{2 k+1}^{\varepsilon}(\bar{R})$. Stabilization has already been taken care of and obviously $\varphi^{!}(1)=0$, so it suffices to check that $A$ and $A \Sigma$ give rise to the same element [W] for every $\Sigma$ in a set of generators of the group $T U^{\varepsilon}(\bar{R})$. If $\Sigma$ lifts to $\tilde{\Sigma}$, then from (2D.1) we have

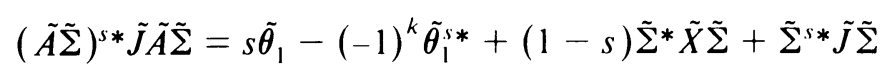

where $\tilde{\theta}_{1}=\tilde{\Sigma} \tilde{\Sigma}^{t *} \tilde{\theta} \tilde{\Sigma}$. Thus, if

$$
\tilde{\Sigma}^{s * \tilde{J} \tilde{\Sigma}}=\tilde{J}+s \tilde{\theta}_{2}-(-1)^{k} \tilde{\theta}_{2}^{s *}
$$

for a suitable $\tilde{\theta}_{2}$, it follows that

$$
\varphi^{\prime}(A \Sigma)=\left[\begin{array}{cc}
\tilde{K} & \tilde{A} \tilde{\Sigma} \\
0 & \tilde{\Sigma}^{*} \tilde{X} \tilde{\Sigma}
\end{array}\right] .
$$

Since

$$
\left(\begin{array}{cc}
1 & 0 \\
0 & \tilde{\Sigma}^{*}
\end{array}\right)\left(\begin{array}{cc}
\tilde{K} & \tilde{A} \\
0 & \tilde{X}
\end{array}\right)\left(\begin{array}{cc}
1 & 0 \\
0 & \tilde{\Sigma}
\end{array}\right)=\left(\begin{array}{cc}
\tilde{K} & \tilde{A} \tilde{\Sigma} \\
0 & \tilde{\Sigma}^{*} \tilde{X} \tilde{\Sigma}
\end{array}\right),
$$

this finishes the proof, provided we can also obtain

(2D.13) $\tilde{\Sigma}$ is a (simple if $\varepsilon=s$ ) isomorphism.

We shall go on to check that (2D.12-13) can be guaranteed for all three kinds of generators of $T U^{\varepsilon}(\bar{R})$.

Case 1.

$$
\Sigma=\left(\begin{array}{cc}
0 & 1 \\
(-1)^{k} & 0
\end{array}\right)
$$

Lift to

$$
\tilde{\Sigma}=\left(\begin{array}{cc}
0 & 1 \\
(-1)^{k} s & 0
\end{array}\right) \quad \text { and take } \quad \tilde{\theta}_{2}=\left(\begin{array}{cc}
0 & 0 \\
(-1)^{k} & 0
\end{array}\right)
$$


Case 2.

$$
\Sigma=\left(\begin{array}{cc}
1 & 0 \\
\nu-(-1)^{k} \nu^{*} & 1
\end{array}\right)
$$

Take

$$
\tilde{\Sigma}=\left(\begin{array}{cc}
1 & 0 \\
s \tilde{\nu}-(-1)^{k} \tilde{\nu}^{s *} & 1
\end{array}\right), \quad \tilde{\theta}_{2}=\left(\begin{array}{cc}
\tilde{\nu} & 0 \\
0 & 0
\end{array}\right)
$$

where $\tilde{\nu}$ covers $\nu$.

Case 3.

$$
\Sigma=\left(\begin{array}{cc}
\left(\sigma^{*}\right)^{-1} & 0 \\
0 & \sigma
\end{array}\right)
$$

where $\sigma$ is a (simple if $\varepsilon=s$ ) isomorphism.

If $\sigma$ lifts to a (simple, if $\varepsilon=s)$ isomorphism $\tilde{\sigma}$ then one takes

$$
\tilde{\Sigma}=\left(\begin{array}{cc}
\left(\tilde{\sigma}^{t *}\right)^{-1} & 0 \\
0 & \tilde{\sigma}
\end{array}\right), \quad \tilde{\theta}_{2}=\left(\begin{array}{ll}
0 & 0 \\
0 & 0
\end{array}\right)
$$

If $\sigma$ does not lift to an isomorphism then (we have $\varepsilon=h$ and) at least $\sigma \oplus \sigma^{-1}$ does. Since we already know that

$$
\varphi^{!}(A)=\varphi^{!}(A \oplus 1)=\varphi^{!}\left((A \oplus 1)\left(\Sigma \oplus \Sigma^{-1}\right)\right)=\varphi^{!}(A \Sigma)+\varphi^{!}\left(\Sigma^{-1}\right),
$$

it suffices to show that $\varphi^{!}\left(\Sigma^{-1}\right)=0$.

If we lift $\Sigma^{-1}$ to $\left(\begin{array}{cc}\tilde{\tau}^{* *} & 0 \\ 0 & \tilde{\rho}\end{array}\right)$ where $\tilde{\rho}$ covers $\sigma^{-1}$ and $\tilde{\tau}$ covers $\sigma$, then we see that $\varphi^{!}\left(\Sigma^{-1}\right)$ is represented by

$$
\left(\begin{array}{cccc}
0 & 1 & \tilde{\tau}^{t *} & 0 \\
(-1)^{k} s & 0 & 0 & \tilde{\rho} \\
0 & 0 & 0 & \tilde{\gamma} \\
0 & 0 & 0 & 0
\end{array}\right) \text { and }\left(\begin{array}{cccc}
0 & 0 & 0 & 0 \\
(-1)^{k}(s-1) & 0 & 0 & \tilde{\rho} \\
(-1)^{k+1} \tilde{\tau}^{t} & 0 & 0 & \tilde{\gamma} \\
0 & 0 & 0 & 0
\end{array}\right)
$$

where $\tilde{\tau} \tilde{\rho}=1+(1-s) \tilde{\gamma}$. From Theorem 1.1 of $[1]$ we see that

$$
A=\left(\begin{array}{cc}
\tilde{\tau} & \tilde{\gamma}^{s} s \\
t-1 & \tilde{\rho}^{t}
\end{array}\right)
$$

is invertible. Hence, so is

$$
B=\left(\begin{array}{ll}
0 & s \\
1 & 0
\end{array}\right) A^{t}\left(\begin{array}{cc}
(-1)^{k+1} & 0 \\
0 & t
\end{array}\right)=\left(\begin{array}{cc}
(-1)^{k}(s-1) & \tilde{\rho} \\
(-1)^{k+1} \tilde{\tau}^{t} & \tilde{\gamma}
\end{array}\right)
$$

But by permuting the basis elements, the above representative for $\varphi^{!}\left(\Sigma^{-1}\right)$ becomes $\left(\begin{array}{ll}0 & 0 \\ B & 0\end{array}\right)$ which represents 0 because

$$
\left(\begin{array}{cc}
0 & 1 \\
\left(B^{*}\right)^{-1} & 0
\end{array}\right)\left(\begin{array}{ll}
0 & 0 \\
B & 0
\end{array}\right)\left(\begin{array}{cc}
0 & B^{-1} \\
1 & 0
\end{array}\right)=\left(\begin{array}{ll}
0 & 1 \\
0 & 0
\end{array}\right) .
$$


3. The main theorem for $l=2 k$. We consider an $S^{1}$-bundle with group $O(2)$, say $p: X \rightarrow Y$, of finite (and simple if $\varepsilon=s$ ) Poincaré complexes. There results a set of fundamental groups and orientation data as in Example 2B.15. Hence, if the formal dimension of $Y$ is $2 k$ we have the algebraic $S^{1}$-transfer map $\varphi^{!}: L_{2 k}^{\varepsilon}\left(\mathbf{Z} \rho ; w_{Y}\right) \rightarrow$ $L_{2 k+1}^{\varepsilon}\left(\mathbf{Z} \pi ; w_{X}\right)$ as in Theorem 2 C.1. We shall prove

MAIN THEOREM, $l$ EVEN. If $\Phi: N^{2 k} \rightarrow Y$ is a surgery problem with pullback $\Psi$ : $M \rightarrow X$, then the surgery obstruction $\sigma(\Psi)$ is given by the formula $\sigma(\Psi)=\varphi^{\prime} \sigma(\Phi)$.

Proof. We can assume that $\Phi$ is $k$-connected and that we have immersed spheres with trivial normal bundles $S_{i}^{k} \times D^{k}(i=1,2, \ldots, r)$ such that the following hold:

(3.1) We have given liftings $\tilde{S}_{i}^{k} \times D^{k}$ in the universal covering of $N$, and the $\tilde{S}_{i}^{k}$ form a $\mathbf{Z} \rho$-basis for $K_{k}(N)$.

(3.2) The spheres $S_{i}^{k} \times 0$ are in general position.

Now a double point $D$ on $\cup_{i} S_{i}^{k} \times 0$ is, by definition, an unordered pair $\{P, Q\}$ with $P \in S_{i}^{k}, Q \in S_{j}^{k}$ (and $P \neq Q$ if $i=j$ ) which coincide in $N$. To each double point $D$, Wall ( $\$ 5$ of [5]) associates a matrix $\alpha_{D} \in M_{r}(\mathbf{Z} \rho)$ which may be described as follows: Order the double point, say with $P$ before $Q$. Then $\alpha_{D}=0$, except for the $(i, j)$ position where it is $\pm g$ for some $g \in \rho$. The group element is determined by the fact that in the universal cover $\tilde{N}, P \in \tilde{S}_{i}^{k} \times 0$ coincides with $g Q \in g\left(\tilde{S}_{j}^{k} \times 0\right)$. Here group elements are identified with covering transformations as usual. The sign is determined by the relation between the given orientation of $\tilde{N}$ at $P$, and the orientation at $P$ coming from $\tilde{S}_{i}^{k} \times 0$ and $g\left(\tilde{S}_{j}^{k} \times 0\right)$ (general position guarantees that these two $k$-dimensional submanifolds "span" the whole $\tilde{N}$ at $P$ ).

Now $\sigma(\Phi)$ is represented by $\alpha=\Sigma \alpha_{D}$ where the sum extends over all double points $D$. We shall see that each double point will give rise to two terms in $\sigma(\Psi)$. To describe $\sigma(\Psi)$ we must first find disjoint framed embedded spheres generating $K_{k}(M)$. The inverse image of the image of $S_{i}^{k} \times D^{k}$ in $M$ is an immersed $S_{i}^{k} \times D^{k}$ $\times S^{1}$. We shall call it $\Sigma_{i}^{k} \times D^{k} \times S^{1}$. If $\{P, Q\}$ is a double point, as above, then $P \times 0 \times S^{1}$ and $Q \times 0 \times S^{i}$ coincide in $M$. If we slide a neighborhood of $P$ along the $S^{1}$-direction we can change the immersion so that:

(3.3) If $\{P, Q\}$ is a double point for $\cup_{i} S_{i}^{k} \times 0$ in $N$, then $(P, 0,0) \in \Sigma_{i}^{k} \times D^{k} \times$ $S^{1}$ coincides with $\left(Q, 0, \frac{1}{2}\right) \in \Sigma_{j}^{k} \times D^{k} \times S^{1}$ (we think of $S^{1}$ as $\mathbf{R} / \mathbf{Z}$ ).

Then for any small $\delta$ we have disjointly embedded framed $k$-spheres $\Sigma_{i}^{k} \times D^{k} \times$ $[-\delta, \delta](i=1,2, \ldots, r)$. An easy application of Gysin sequences reveals that

(3.4) $K_{k}(M)$ is generated by the spheres $\Sigma_{i}^{k} \times 0 \times 0, i=1,2, \ldots, r$, over $\mathbf{Z} \pi$.

Following $\$ 6$ of Wall's book [5], we let $U=\cup_{i} \Sigma_{i}^{k} \times D^{k} \times[-\delta, \delta], M_{0}=M-$ $\operatorname{int}(U)$, and $\partial U=\cup_{i} \Sigma_{i}^{k} \times \partial\left(D^{k} \times[-\delta, \delta]\right)$. Then $K_{k+1}\left(M_{0}, \partial U\right)$ is a free $\mathbf{Z} \pi$-module of rank $r$ (with a preferred basis if $\varepsilon=s$ ). Also, $K_{k}(\partial U)$ is free with a basis consisting of $e_{i}=\Sigma_{i}^{k} \times 0 \times \delta(i=1,2, \ldots, r)$ and $f_{i}=p_{i} \times \partial\left(D^{k} \times[-\delta, \delta]\right)(i=$ $1,2, \ldots, r)$ where $p_{i} \in \Sigma_{i}^{k}$ is arbitrary. And

(3.5) $\sigma(\Psi)$ is represented by the matrix $\left(\begin{array}{c}\eta \\ \beta\end{array}\right)$ of $\partial: K_{k+1}\left(M_{0}, \partial U\right) \rightarrow K_{k}(\partial U)$ (with respect to the preferred basis if $\varepsilon=s$ ).

In fact, Wall's description is the following: Identify $K_{k}(\partial U)$ with a standard kernel $H_{r}$ using the basis above. Identify $K_{k+1}\left(M_{0}, \partial U\right)$ and $K_{k+1}(U, \partial U)$ with 
subkernels in $H_{r}$ using the boundary maps. Then $\sigma(\Psi)$ is represented by any unitary matrix $\left(\begin{array}{l}* \eta \\ * \beta\end{array}\right)$ which maps $K_{k+1}(U, \partial U)$ (simply) onto $K_{k+1}\left(M_{0}, \partial U\right)$. Since $K_{k+1}(U, \partial U)$ has basis $f_{1}, \ldots, f_{r}$, this means that $\left(\begin{array}{c}\eta \\ \beta\end{array}\right)$ becomes the matrix of $\partial$ : $K_{k+1}\left(M_{0}, \partial U\right) \rightarrow K_{k}(\partial U)$ as claimed.

REMARK. Apparently Wall thinks of $K_{k+1}(U, \partial U)$ as having $e_{1}, \ldots, e_{r}$ as a basis. Then he defines $T U_{r}(R)$ to be those automorphisms which leave $\operatorname{span}\left\{e_{i}\right\}$ invariant (and induce a simple isomorphism on it if $\varepsilon=s$ ), whereas we have required that $\operatorname{span}\left\{f_{i}\right\}$ be fixed. Compare also his formula at the top of his p. 58 with our (2A.13).

In view of (3.5) we now go searching for a (preferred, if $\varepsilon=s$ ) basis for $K_{k+1}\left(M_{0}, \partial U\right)$ which is isomorphic to $K_{k+1}(M, U)$ under excision. Since we have to compute boundaries, we prefer to represent homology classes where they really exist, i.e. in the universal covers. It is easily seen that we have a commutative diagram

$$
\begin{array}{ccccc}
\bar{S}^{1} & \rightarrow & S^{1} & = & S^{1} \\
\downarrow & & \downarrow & & \downarrow \\
\tilde{M} & \rightarrow & \bar{M} & \rightarrow & M \\
\downarrow & & \downarrow & & \downarrow \\
\tilde{N} & = & \tilde{N} & \rightarrow & N
\end{array}
$$

where $\bar{M}$ is the pullback of $M$ over $\tilde{N}, \tilde{M}$ is the universal covering and $\tilde{M} \rightarrow \tilde{N}$ is a principal $\bar{S}^{1}$-bundle. Here $\bar{S}^{1}=\mathbf{R}$ if $\pi_{1}\left(S^{1}\right) \rightarrow \pi_{1}(M)$ is monic and $\bar{S}^{1}=\mathbf{R} / n \mathbf{Z}$ if the image of $\pi_{1}\left(S^{1}\right)$ has order $n$. The map $\bar{S}^{1} \rightarrow S^{1}$ is the standard covering of degree $n \leqslant \infty$.

We fix the generator for $\pi_{1}\left(S^{1}\right)$ in the usual way. Then its image $t \in \pi_{1}(M)$, viewed as a covering transformation, coincides with the action of $1 \in \bar{S}^{1}$ in $\tilde{M}$. Also, if $\tilde{g} \in \pi=\pi_{1}(M)$ is any covering transformation and $z \in \bar{S}^{1}$, then

$$
z \tilde{g}(x)=\tilde{g}(\omega(\varphi(\tilde{g})) z)(x) .
$$

The immersed $\tilde{S}_{i}^{k} \times D^{k}$ in $\tilde{N}$ and $\Sigma_{i}^{k} \times D^{k} \times S^{1}$ in $M$ are covered in $\tilde{M}$ by an immersed $S_{i}^{k} \times D^{k} \times \bar{S}^{1}$ which we call $\tilde{\Sigma}_{i}^{k} \times D^{k} \times \bar{S}^{1}$. Since unit translations in the $\bar{S}^{1}$-direction correspond to applications of $t \in \pi$, we see that:

(3.7) If the (ordered) double point $\{P, Q\}$ has $P=g Q$ in $\tilde{N}$, then there is a unique $\tilde{g} \in \pi$ which maps to $g$ in $\rho$, and for which $(P, 0,0) \in \tilde{\Sigma}_{i}^{k} \times D^{k} \times \bar{S}^{1}$ coincides with $\tilde{g}\left(Q, 0, \frac{1}{2}\right) \in \tilde{g}\left(\tilde{\Sigma}_{j}^{k} \times D^{k} \times \bar{S}^{1}\right)$. Consequently, $(P, 0, z)$ coincides with $\tilde{g}\left(Q, 0, \frac{1}{2}+\omega(g) z\right)$.

It is clear that the singular cylinders $\tilde{\Sigma}_{i}^{k} \times 0 \times[\delta, 1-\delta]$ represent elements in $K_{k+1}(M, U)$. Since one also has geometric generators for $K_{k+1}(M)$ (namely $\tilde{\Sigma}_{i}^{k} \times 0$ $\left.\times \bar{S}^{1}\right)$ and for $K_{k}(M)$ (namely $\left.\tilde{\Sigma}_{i}^{k} \times 0 \times 0\right)$ one can compare the exact sequence

$$
0 \rightarrow K_{k+1}(M) \rightarrow K_{k+1}(M, U) \rightarrow K_{k}(U) \rightarrow K_{k}(M) \rightarrow 0
$$

to the direct sum of $r$ copies of the algebraic exact sequence

$$
0 \rightarrow \mathbf{Z} \rho \stackrel{T}{\rightarrow} \mathbf{Z} \pi \stackrel{t-1}{\rightarrow} \mathbf{Z} \pi \rightarrow \mathbf{Z} \rho \rightarrow 0
$$

(where $T=1+t+\cdots+t^{n-1}$ ). The five lemma then implies:

(3.8) The singular cylinders $C_{j}=\tilde{\Sigma}_{j}^{k} \times 0 \times[\delta, 1-\delta]$ form a $\mathbf{Z} \pi$ basis for $K_{k+1}(M, U)$. 
REmarks. (1) If $n=\infty$ there are slight modifications: $K_{k+1}(M)=0$ and $t-1$ : $\mathbf{Z} \pi \rightarrow \mathbf{Z} \pi$ is monic.

(2) If $\varepsilon=s$ we need to know that the above basis is a preferred one. This follows easily from the explicit geometric nature of the basis.

Now $C_{j}$ has two obvious boundary components, namely $\tilde{\Sigma}_{j}^{k} \times 0 \times(1-\delta)$ which is homologous in $\partial U$ to $e_{j} t^{-1}=t\left(\tilde{\Sigma}_{j}^{k} \times 0 \times \delta\right)=\tilde{\Sigma}_{j}^{k} \times 0 \times(1+\delta)$, and $\tilde{\Sigma}_{j}^{k} \times 0 \times$ $\delta=e_{j}$. Hence, the component of $\partial C_{j}$, after the basis elements $\left\{e_{i}\right\}$, is $e_{j}(-1)^{k}\left(t^{-1}-1\right)$. If $E^{-1}: K_{k+1}\left(M_{0}, \partial U\right) \rightarrow K_{k+1}(M, U)$ is the excision isomorphism, then $\partial\left(E C_{j}\right)$ contains the same term, but it also contains terms involving the $f_{i}$. In fact, the excision creates a boundary component of type $f_{i}$ whenever the cylinder $\tilde{\Sigma}_{j}^{k} \times 0 \times$ $[\delta, 1-\delta]$ is intersected by some component $\tilde{h}\left(\tilde{\Sigma}_{i}^{k} \times D^{k} \times[-\delta, \delta]\right)$ of $\tilde{U}$. Each double point gives rise to two such intersections (with $i$ and $j$ interchanged in one of them). Let the double point $\{P, Q\}$ give rise to $\alpha_{D}=e_{i j}(\varepsilon g)$ when ordered with $P$ first $(\varepsilon= \pm 1)$. By (3.7), $\left(Q, 0, \frac{1}{2}+z\right) \in \tilde{\Sigma}_{j}^{k} \times D^{k} \times \bar{S}^{1}$ coincides with $\tilde{g}^{-1}(P, 0, \omega(g) z) \in \tilde{g}^{-1}\left(\tilde{\Sigma}_{i}^{k} \times D^{k} \times \bar{S}^{1}\right)$ for a unique $\tilde{g}$ above $g$. Consequently, $C_{j}$ is intersected by $\tilde{g}^{-1}\left(\tilde{\Sigma}_{i}^{k} \times D^{k} \times[-\delta, \delta]\right)$ in $\tilde{g}^{-1}\left(P \times D^{k}\right) \times\left[\frac{1}{2}-\delta, \frac{1}{2}+\delta\right] \subseteq \tilde{\Sigma}_{j}^{k} \times 0 \times$ $[\delta, 1-\delta]$. The orientation of $\left[\frac{1}{2}-\delta, \frac{1}{2}+\delta\right]$ is $\omega(g)$ times that of $[\delta, 1-\delta]$, and the orientation of $\tilde{g}^{-1}\left(P \times D^{k}\right)$ viz-a-viz $\tilde{\Sigma}_{j}^{k}$ is the same as that of $g^{-1}\left(P \times D^{k}\right)$ viz-a-viz $\tilde{S}_{j}^{k}($ in $\tilde{N})$.

Here we know that $\tilde{S}_{i}^{k}$ and $g \tilde{S}_{j}^{k}$, together, give the $\varepsilon$-orientation at $P$. Hence, $g^{-1}\left(\tilde{S}_{i}^{k}\right)$ and $\tilde{S}_{j}^{k}$ give the $\varepsilon w_{B}(g)$-orientation at $Q$. But also, $g^{-1}\left(\tilde{S}_{i}\right)$ and $g^{-1}\left(P \times D^{k}\right)$ give the $w_{B}(g)$-orientation at $Q$. It follows that the orientation of $g^{-1}\left(P \times D^{k}\right)$ and $\tilde{S}_{j}^{k}$ at $Q$ differ by $\varepsilon$ and we get:

(3.9) If the double point $\{P, Q\}$, with $P$ put first, contributes $e_{i j}(\varepsilon g)$ to $\alpha$ then it contributes $e_{i j}(\varepsilon \omega(g) \tilde{g})$ to $\beta$.

Now consider $\{P, Q\}$ with $Q$ put first. Its contribution to $\alpha$ is well known to be $e_{j i}\left(\varepsilon(-1)^{k} w_{B}(g) g^{-1}\right)$. Hence, by (3.9) its contribution to $\beta$ is of the form $e_{j i}\left(\varepsilon(-1)^{k} w_{E}(\tilde{g}) \widetilde{g^{-1}}\right)$ where $g^{-1}$ is some lift of $g^{-1}$. However, we still have to determine the relationship between the lifts of $g$ and $g^{-1}$. In (3.9), $\tilde{g}$ is determined by (3.7), i.e. by the equation $(P, 0,0)=\tilde{g}\left(Q, 0, \frac{1}{2}\right)$. When we interchange $P$ and $Q$ and remember (3.6), this becomes

$$
(Q, 0,0)=\left\{\begin{array}{cc}
(t \tilde{g})^{-1}\left(P, 0, \frac{1}{2}\right), & \omega(g)=+1, \\
\tilde{g}^{-1}\left(P, 0, \frac{1}{2}\right), & \omega(g)=-1 .
\end{array}\right.
$$

This determines $\widetilde{g^{-1}}$ as $\omega(g) s\left(\tilde{g}^{t}\right)^{-1}$, and the contribution from $D$ to $\beta$ becomes

$$
\beta_{D}=\varepsilon \omega(g)\left(e_{i j}(\tilde{g})+(-1)^{k} e_{j i}\left(s \tilde{g}^{t *}\right)\right)
$$

If we lift $\alpha=\Sigma_{D} e_{i j}(\varepsilon g)$ to $\tilde{\alpha}=\Sigma_{D} e_{i j}\left(\varepsilon \omega(g) \tilde{g}^{s}\right)$, we get the desired formula, $\beta=\tilde{\alpha}^{t}+(-1)^{k} \tilde{\alpha}^{*} s$.

REMARK. In (3.3) one could slide the neighborhood of $P$ some extra full turns around in the $S^{1}$-direction. This would replace $\tilde{g}$ by $\tilde{g} t^{i}$ for some $i$. In this way any lifting $\tilde{\alpha}$ of $\alpha$ can be geometrically realized. Hence $\S 3$ actually constitutes a full proof (without reference to $\S 2$ ) of the Main Theorem (for $l=2 k$ ). 
4. The main theorem for $l=2 k+1$. The situation is exactly as in $\S 3$, i.e. we have a pullback diagram

$$
\begin{array}{ccc}
\Psi: M & \rightarrow & X \\
\downarrow & & \downarrow \\
\Phi: N & \rightarrow & Y
\end{array}
$$

of $S^{1}$-bundles; $N \rightarrow Y, \nu_{N} \rightarrow \xi$ is a surgery problem of dimension $2 k+1$, and we have the algebraic $S^{1}$-transfer $\varphi^{!}: L_{2 k+1}^{\varepsilon}\left(\mathbf{Z} \rho ; w_{Y}\right) \rightarrow L_{2 k+2}^{\varepsilon}\left(\mathbf{Z} \pi ; w_{X}\right)$ from Theorem 2D.2.

MAIN THEOREM, $l$ ODD. The surgery obstructions $\sigma(\Phi)$ and $\sigma(\Psi)$ are related by the formula $\sigma(\Psi)=\varphi^{!}(\sigma(\Phi))$.

By $\S 6$ of [5] we have embedded, framed spheres $S_{i}^{k} \times D_{i}^{k+1}$ in $N(i=1,2, \ldots, r)$ such that the spheres $S_{i}^{k} \times 0$ (with a chosen path to the basepoint of $N$ ) form a basis for the $\mathbf{Z} \rho$-module $K_{k}(N)$. Also letting $U=\cup_{i} S_{i}^{k} \times D_{i}^{k+1}, N_{0}=N \backslash \operatorname{int}(U)$, and $\partial U=\cup_{i} S_{i}^{k} \times S_{i}^{k}$, the surgery obstruction $\sigma(\Phi)$ is represented by the matrix $\left(\begin{array}{c}\eta \\ \beta\end{array}\right)$ of $\partial: K_{k+1}\left(N_{0}, \partial U\right) \rightarrow K_{k}(\partial U)$ with respect to a (preferred if $\varepsilon=s$ ) basis for $K_{k+1}\left(N_{0}, \partial U\right)$ and the obvious basis for $K_{k}(\partial U)$. The latter is geometrically represented by the spheres $S_{i}^{k} \times p_{i}, q_{i} \times S_{i}^{k}$ along with paths $\sigma_{i}$ from $q_{i} \times p_{i}$ to the basepoint of $N$. (The orientation of $S_{i}^{k} \times D_{i}^{k+1}$ is chosen to coincide with the orientation of $N$ at the basepoint when transported along $\sigma_{i \cdot}$.)

4A. Geometric generators for $K_{k+1}\left(N_{0}, \partial U\right)$. Let $\Gamma^{k+1}$ be a "sphere with holes", i.e. a $(k+1)$-dimensional sphere with a number of disjoint $(k+1)$-disks removed. Hence, $\partial \Gamma^{k+1}$ is a union of $k$-spheres, and if $f:\left(\Gamma^{k+1}, \partial \Gamma\right) \rightarrow\left(N_{0}, \partial U\right)$ is any continuous map while $\gamma$ is a path from $f\left(\Gamma^{k+1}\right)$ to the basepoint, then these data specify a unique element of $H_{k+1}\left(N_{0}, U\right)$ (i.e. really of $H_{k+1}\left(\tilde{N}_{0}, \tilde{U}\right)$ where $\tilde{N}_{0}$ is the restriction of the universal covering $\tilde{N}$ to $N_{0}$ ). It seems natural to consider the following

ConjeCture. $K_{k+1}\left(N_{0}, \partial U\right)$ has a (preferred, if $\varepsilon=s$ ) basis consisting of $r$ immersed "spheres with holes" $\Gamma_{i}, i=1,2, \ldots, r$, in general position. The self-intersections of $\cup \Gamma_{i}$ is a set of paths with endpoints on $\partial\left(\cup_{i} \Gamma_{i}\right)$, and each boundary component of each $\Gamma_{i}$ is embedded as a $S_{j}^{k} \times\{p t\}$ or as a $\{p t\} \times S_{j}^{k}$.

In this subsection we prove that it suffices to consider the case when the conjecture holds.

In fact, by [2], the geometrically defined transfer homomorphism $L_{2 k+1}^{\varepsilon}\left(\mathbf{Z} \rho ; w_{Y}\right)$ $\rightarrow L_{2 k+2}^{\varepsilon}\left(\mathrm{Z} \pi ; w_{X}\right)$ depends only on the fundamental group and orientation data. Thus, it suffices to prove the theorem for suitably chosen examples. We use Wall's realization theorem (Theorem 6.5 of [5]) to produce such examples. First of all, there exist a manifold $W$ of dimension $2 k$ and a map $W \rightarrow Y$ which induces an isomorphism on $\pi_{1}$ and an epimorphism on $\pi_{2}$. We replace $S^{1} \rightarrow X \rightarrow Y$ by its pullback over $W \times I$ via $W \times I \rightarrow W \rightarrow Y$ which does have the same fundamental group and orientation data. To avoid excessive notation let us assume $Y=W \times I$.

By the theorem of Wall quoted above any element $\left[\begin{array}{l}\eta \\ \beta\end{array}\right] \in L_{2 k+1}^{\varepsilon}\left(\rho ; w_{Y}\right)$ can be obtained as the surgery obstruction of a map $\Phi: N \rightarrow Y$. We proceed to recall how $\Phi: N \rightarrow Y$ is constructed. 
We perform surgery on $1_{W}: W \rightarrow W$ to kill $r$ trivial $(k-1)$-spheres, thereby replacing $W \times I$ by its boundary connected sum with $r$ copies of $S^{k} \times D^{k+1}$, and $W$ by its connected sum with $r$ copies of $S^{k} \times S^{k}$.

(4A.1) The resulting kernel $K_{k}$ is the sum of $r$ standard planes. After choice of a path from each $S^{k} \times S^{k}$ to the basepoint a basis for $K_{k}$ is given as $e_{i}=S_{i}^{k} \times p t$ $(i=1,2, \ldots, r), f_{i}=p t \times S_{i}^{k}(i=1,2, \ldots, r)$.

We think of the matrix $\left(\begin{array}{c}\eta \\ \beta\end{array}\right)$ as the $r$ elements

$$
\zeta_{j}=\sum_{i} e_{i} \eta_{i j}+\sum_{i} f_{i} \beta_{i j}
$$

in the above kernel. For any $g \in \rho, e_{i} g$ is represented by $S_{i}^{k} \times p t$ with its original path to the basepoint followed by a loop representing $g$. Similarly, $f_{i} h$ is represented in terms of $p t \times S_{i}^{k}$ and its path to the basepoint. Hence,

(4A.3) $\zeta_{j}$ is represented by a disjoint collection of $S_{i}^{k} \times p t$ and $p t \times S_{i}^{k}$, together with paths to the basepoint. $S_{i}^{k} \times p t$ (or $p t \times S_{i}^{k}$ ) has the standard orientation if the corresponding term $e_{i} g$ (or $f_{i} h$ ) appears with a + in (4A.2), otherwise it has the opposite orientation.

We wish to represent each $\zeta_{j}$ by one immersed sphere. This is obtained by the addition described in $\$ 5$ of [5], i.e.:

(4A.4) Tubing up the various spheres which appear in (4A.3), along disjoint tubes homotopic to the path from one sphere to the basepoint followed by the path from the basepoint to the other sphere and matching orientations, we get $\zeta_{j}$ represented by an immersed sphere $Z_{j}$ with a path to the basepoint.

As in [5] we see that surgery is possible on this collection of $k$-spheres, i.e. there is a regular homotopy $Z_{j}(t), 0 \leqslant t \leqslant 1$, of the $Z_{j}$ 's so that the $Z_{j}(1)$ 's are disjointly embedded. When we perform the surgery, the result is mapped to $W$ by a simple homotopy equivalence, and the trace $N$ of the surgery with its map $\Phi$ to $Y$ represents $\left[\begin{array}{l}\eta \\ \beta\end{array}\right]$.

In this surgery problem, $K_{k+1}\left(N_{0}, \partial U\right)$ has a basis consisting of the cores of the handles attached, i.e. consisting of embedded $(k+1)$-disks with boundaries $Z_{j}(1)$. We now extend $Z_{j}(1-t), 0 \leqslant t \leqslant 1$, to a regular homotopy of all of $N_{0}$ by using a collar of the boundary to taper it down to the trivial regular homotopy. This replaces the basis disks by immersed disks $\Gamma_{j}^{\prime}$ where $\partial \Gamma_{j}^{\prime}$ is immersed as $Z_{j}=Z_{j}(0)$.

The regular homotopy $Z_{j}(t)$ really came from doing a number of Whitney tricks. When we undo these and taper off the undoing in a collar we create double point paths but no other singularities (see Figure 1).

We finally "fill in" the tubes that were used in (4A.4). This changes $\Gamma_{j}^{\prime}$ to the required $\Gamma_{j}$.
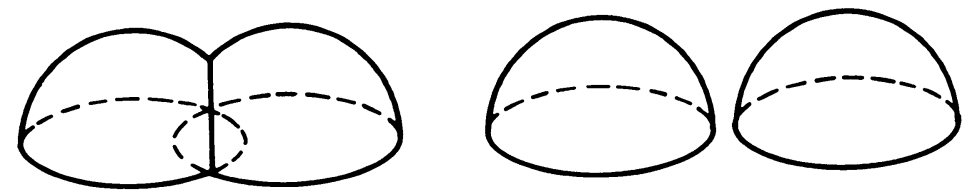

FIGURE 1

4B. Analysis of double point paths. Each boundary component of $\Gamma_{j}$ is labelled as $\varepsilon e_{i} g$ (or $\varepsilon f_{i} h$ ); $\varepsilon$ indicates the orientation inherited from $\Gamma_{j}$ in comparison to the one 
coming from $S_{i}^{k} \times D^{k+1}$, and $g$ is determined by the path $\sigma_{i}$ from the basepoint to $S_{i}^{k} \times D^{k+1}$, through $\Gamma_{j}$ to its basepoint, and back to the basepoint via the path $\gamma_{j}$ of $\Gamma_{j}$.

Now consider a double point path $D$ for $\Gamma_{*}=\Gamma_{1} \cup \Gamma_{2} \cup \cdots \cup \Gamma_{r}$. Let the two endpoints be the intersection of $\varepsilon_{1} e_{i_{1}} g_{1}$ and $\varepsilon_{2} f_{i_{1}} h_{1}$, and the intersection of $\varepsilon_{3} e_{i_{2}} g_{2}, \varepsilon_{4} f_{i_{2}} h_{2}$, respectively. Call $D$ of type I if (in the original) $\varepsilon_{1} e_{i_{1}} g_{1}$ is joined to $\varepsilon_{3} e_{i_{2}} g_{2}$ (and consequently, $\varepsilon_{2} f_{i_{1}} h_{1}$ is joined to $\varepsilon_{4} f_{i_{2}} h_{2}$ ). Call it of type II if $\varepsilon_{1} e_{i_{1}} g_{1}$ is joined to $\varepsilon_{4} f_{i_{2}} h_{2}$ (and $\varepsilon_{2} f_{i_{1}} h_{1}$ to $\varepsilon_{3} e_{i_{2}} g_{2}$ ). Finally, let $(j, k)$ be determined by the fact that the intersection occurs between $\Gamma_{j}$ and $\Gamma_{k}$ (thus $\varepsilon_{1} e_{i}, g_{1} \subseteq \Gamma_{j}$ ).

If $D$ is of type $\mathrm{I}$, then $g_{1}=\left[\sigma_{i_{1}}^{-1} \tau_{j}^{\prime} \gamma_{j}\right]$ and $g_{2}=\left[\sigma_{i_{1}}^{-1} \tau_{j}^{\prime \prime} \gamma_{j}\right]$ with $\tau_{j}^{\prime}, \tau_{j}^{\prime \prime}$ paths in $\Gamma_{j}$ (recall that $\sigma_{i}$ is the path from $S_{i}^{k} \times S_{i}^{k}$ and $\gamma_{j}$ the one from $\Gamma_{j}$ ). Similarly, $h_{1}=\left[\sigma_{i_{1}}^{-1} \tau_{k}^{\prime} \gamma_{k}\right]$ and $h_{2}=\left[\sigma_{i_{2}}^{-1} \tau_{k}^{\prime \prime} \gamma_{k}\right]$. It follows that $g_{1}^{-1} h_{1}=g_{2}^{-1} h_{2}$ (the triangles $\tau_{j}^{\prime} \tau_{j}^{\prime \prime} D$ and $\tau_{k}^{\prime} \tau_{k}^{\prime \prime} D$ can be filled in inside $Z_{j}$ ).

In case of a double point path of type II, a similar analysis reveals that $g_{1}^{-1} h_{1}=h_{2}^{-1} g_{2}$.

One can also analyse the orientation matters for a double point path $D$. The results are

$$
\begin{array}{ll}
\varepsilon_{1} \varepsilon_{2} w_{B}\left(g_{1}\right)=-\varepsilon_{3} \varepsilon_{4} w_{B}\left(g_{2}\right) & \text { if } D \text { is of type I, } \\
\varepsilon_{1} \varepsilon_{2} w_{B}\left(g_{1}\right)=-(-1)^{k} \varepsilon_{3} \varepsilon_{4} w_{B}\left(h_{2}\right) & \text { if } D \text { is of type II. }
\end{array}
$$

Recalling that $g^{*}=w_{B}(g) g^{-1}$, we see that the above can be summarized in:

(4B.2) If a double point path $D$ is of type I, then $\varepsilon_{1} \varepsilon_{2} g_{1}^{*} h_{1}=-\varepsilon_{3} \varepsilon_{4} g_{2}^{*} h_{2}$. If it is of type II, then $\varepsilon_{1} \varepsilon_{2} g_{1}^{*} h_{1}=-(-1)^{k} \varepsilon_{3} \varepsilon_{4} h_{2}^{*} g_{2}$.

By $(4 A .2,4), \partial Z_{j}=\sum_{i} e_{i} \eta_{i j}+\sum_{i} f_{i} \beta_{i j}$. Also let $\mathscr{D}_{\mathrm{I}}$, respectively $\mathscr{D}_{\mathrm{II}}$, be the set of double point paths of type I, respectively II. To each $D \in \mathscr{D}_{\text {II }}$, we associate a matrix $z_{D}=e_{j k}\left(\varepsilon_{1} \varepsilon_{2} w_{B}\left(g_{1}\right) g_{1}^{-1} h_{1}\right)$. We then claim

$$
\eta^{*} \beta=\sum_{D \in \mathbb{D Q D}_{11}}\left(z_{D}-(-1)^{k} z_{D}^{*}\right) .
$$

The proof is easy: Recall that $e_{i j}(g)$ is the $r \times r$ matrix with $g$ in position $(i, j)$ as the only nonzero entry. Terms of the form $e_{i j}( \pm g)$ in $\eta$, respectively $\beta$, correspond bijectively to boundary components of type $S_{i}^{k} \times p t$, respectively of type $p t \times S_{i}^{k}$, in $\partial Z_{j}$. Terms in $\eta^{*} \beta$ correspond bijectively to self-intersections of $\cup_{j} \partial Z_{j}$. These come in pairs (determined by double point paths). By (4B.1) the contribution of the two ends of a path of type I cancel in $\eta^{*} \beta$. And the terms from the two ends of a path $D \in \mathscr{Q}_{\text {II }}$ have the form $z_{D}-(-1)^{k} z_{D}^{*}$.

4C. Surgery below the middle dimension in the total space. The spheres $E_{i}, F_{i}$.

We consider the $S^{1}$-bundle trivialized when restricted to $U=\cup_{i=1}^{r} S_{i}^{k} \times D^{k+1} \subset$ $N$. Thus, we have the diagram:

$\begin{array}{cccccc} & & S^{1} & = & S^{1} \\ & & \downarrow & & \downarrow \\ U \times S^{1} & \subset & M & \rightarrow & X \\ \downarrow & & \downarrow & & \downarrow \\ U & \subset & N & \rightarrow & Y\end{array}$


Also, recall that we take $S^{1}=\mathbf{R} / \mathbf{Z}$. We perform surgery on each $S_{i}^{k} \times D^{k+1} \times\left[-\frac{1}{4}\right.$, $\left.\frac{1}{4}\right]$. The resulting surgery problem $M^{\prime} \rightarrow X$ has $M^{\prime}=M_{0} \cup V^{\prime}$ where $M_{0}=p^{-1}\left(N_{0}\right)$, $N_{0}=N-\operatorname{int}(U)$, and $V^{\prime}$ is the disjoint union for $i=1,2, \ldots, r$ of

$$
\begin{aligned}
V_{i}^{\prime}= & \left(S_{i}^{k} \times D_{i}^{k+1} \times\left[\frac{1}{4}, \frac{3}{4}\right]\right) \cup\left(D_{i}^{k+1} \times D_{i}^{k+1} \times\left\{\frac{3}{4}\right\}\right) \\
& \cup\left((-1)^{k} D_{i}^{k+1} \times S_{i}^{k} \times\left[-\frac{1}{4}, \frac{1}{4}\right]\right) \\
& \cup\left(-D_{i}^{k+1} \times D_{i}^{k+1} \times\left\{\frac{1}{4}\right\}\right) .
\end{aligned}
$$

We choose paths $\tilde{\sigma}_{i}$ from $S_{i}^{k} \times D_{i}^{k+1} \times\left\{\frac{3}{4}\right\}$ to the basepoint of $M$ covering $\sigma_{i}$ in $N$. And we orient the embeddings so that the product orientation on $S_{i}^{k} \times D_{i}^{k+1} \times S^{1}$, transported to $*$ along $\tilde{\sigma}_{i}$, gives the chosen standard orientation of $M$ there. The signs in the above description of $V_{i}^{\prime}$ indicate how the various pieces of $V_{i}^{\prime}$ must be oriented in order to make $V_{i}^{\prime}$ oriented (and contain $S_{i}^{k} \times D_{i}^{k+1} \times\left[\frac{1}{4}, \frac{3}{4}\right]$ as a positive piece).

Since $K_{k}(N)$ is generated by the $S_{i}^{k} \times 0$, it easily follows that $K_{k}(M)$ is generated by $S_{i}^{k} \times 0 \times 0$ (use a Gysin sequence). Thus, $\Psi^{\prime}: M^{\prime} \rightarrow X$ is $k$-connected, and to compute $\sigma(\Psi)$ we just need to find a set of spheres immersed in $M$ which generate $K_{k+1}\left(M^{\prime}\right)$ and compute their (self- and mutual) intersections.

Using the above orientation conventions we see that there are some obvious embedded spheres in the kernel of $\Psi^{\prime}: M^{\prime} \rightarrow X$, namely

$$
\begin{aligned}
E_{i}= & (-1)^{k}\left(0 \times D_{i}^{k+1} \times\left\{-\frac{1}{4}\right\}\right) \cup\left(0 \times S_{i}^{k} \times\left[-\frac{1}{4}, \frac{1}{4}\right]\right) \\
& \cup(-1)^{k+1}\left(0 \times D_{i}^{k+1} \times\left\{\frac{1}{4}\right\}\right), \\
F_{i}= & (-1)^{k}\left(D_{i}^{k+1} \times 0 \times\left\{\frac{1}{4}\right\}\right) \cup(-1)^{k}\left(S_{i}^{k} \times 0 \times\left[\frac{1}{4}, \frac{3}{4}\right]\right) \\
& \cup\left(-D^{k+1} \times 0 \times\left\{\frac{3}{4}\right\}\right)
\end{aligned}
$$

$(i=1,2, \ldots, r)$.

Clearly, $E_{i}$ and $F_{i}$ intersect transversely at $\left(0,0, \frac{1}{4}\right)$ and $\left(0,0, \frac{3}{4}\right)$ and an easy computation - using the above path $\tilde{\sigma}_{i}$ and the above orientation conventions-shows that

$$
E_{i} \cdot F_{i}=1-t
$$

4D. Liftings of the "spheres with holes" to $M$; the spheres $G_{j}$. We view the immersed "spheres with holes" $\Gamma_{j}$ as immersions $\Gamma_{j}: \Sigma_{j} \rightarrow N_{0}$ where $\Sigma_{j}$ is a nonsingular sphere with an adequate number of disks removed. Let $M_{0}=p^{-1}\left(N_{0}\right)$ and recall that $\partial M_{0}=\cup_{i} S_{i}^{k} \times S_{i}^{k} \times S^{1}$ under the trivialization from $\S 4 \mathrm{C}$.

(4D.1) We can choose a lifting

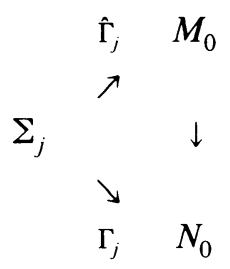

such that each boundary component of the form $S_{i}^{k} \times p t$ (respectively $p t \times S_{i}^{k}$ ) is lifted to $S_{i}^{k} \times p t \times 0$ (respectively $p t \times S_{i}^{k} \times \frac{1}{2}$ ). 
The proof is easy: Since surgery was done only above $U$, we still have an $S^{1}$-bundle above $N_{0}$. It becomes trivial when pulled back to $\Sigma_{j}$, so some lifting $\hat{\Gamma}_{j}$ certainly exists. To obtain the extra conditions on the boundary we use such an arbitrary lifting $\hat{\Gamma}_{j}$ only outside a collar $\partial \Sigma_{j} \times[0,1]$ of the boundary $\partial \Sigma_{j}$. We may assume that $\Gamma_{j}$ maps this collar, in a collar preserving way, to a collar of $\partial U=\cup S_{i}^{k}$ $\times S_{i}^{k}$. Thus on any one component $S^{k} \times[0,1]$ of $\partial \Sigma_{j} \times[0,1]$, one has $\Gamma_{j}(x, t)=$ $(x, q, t)$ (or $\left.\Gamma_{j}(x, t)=(q, x, t)\right)$ for a fixed $q$. At one end of the collar one then has $\hat{\Gamma}_{j}(x, 0)=(x, q, 0, f(x))\left(\right.$ or $\left.\hat{\Gamma}_{j}(x, 0)=(q, x, 0, f(x))\right)$ for some map $f: S^{k} \rightarrow S^{1}$. Let $f_{t}$ be a homotopy from $f=f_{0}$ to $f_{1}$, the constant map with value 0 (or $\frac{1}{2}$ ) in $\mathbf{R} / \mathbf{Z}=S^{1}$. Then $\hat{\Gamma}_{j}(x, t)=\left(x, q, t, f_{t}(x)\right)\left(\right.$ or $\left.\hat{\Gamma}_{j}(x, t)=\left(q, x, t, f_{t}(x)\right)\right)$ is a lift of $\Gamma_{j}$ on the collar which produces the desired result. It is then clear that:

(4D.2) One gets immersed spheres $G_{j}: S^{k+1} \rightarrow M^{\prime}$ by capping off the holes in $\Gamma_{j}$ and extending the map $\hat{\Gamma}_{j}: \Sigma_{j} \rightarrow M_{0}$ by $D_{i}^{k+1} \times q \times 0$ or $q \times D_{i}^{k+1} \times \frac{1}{2}$.

For each one of the immersed spheres so constructed we choose a path $\hat{\gamma}_{j}$ to basepoint covering the path $\gamma_{j}$ from $\Gamma_{j}$ to basepoint. Then $G_{j}$ represents a homology class in $K_{k+1}\left(M^{\prime}\right)$. Clearly, the sphere $E_{i}$ intersects $G_{j}$ precisely in one point for each capped-off boundary component $D_{i}^{k+1} \times q \times 0$. The intersection point is counted with a group element $\tilde{g} \in \pi$ given as $\tilde{g}=\left[\tilde{\sigma}_{i} \cdot \tilde{\tau} \cdot \tilde{\gamma}_{j}\right]$ where $\tilde{\tau}$ is a path in $\hat{\Gamma}_{j}$. It follows that $\tilde{g}$ covers the group element $g \in \rho$ assigned to the boundary component in question. Also the orientations have been chosen to make the sign of the intersection point fit with that of the boundary component. Hence, we get

(4D.3) $E_{*} \cdot G_{*}=\tilde{\eta}, F_{*} \cdot G_{*}=\tilde{\beta}$ where $\tilde{\eta}, \tilde{\beta}$ are $r \times r$ matrices over $\mathbf{Z} \pi$ with $\varphi(\tilde{\eta})=\eta, \varphi(\tilde{\beta})=\beta$.

When we come to compare the algebra with the geometry, the algebra dictates the extra condition that $(\underset{\tilde{\beta}}{\tilde{q}})$ is part of an invertible matrix as in Proposition 2D.11 (see also Lemma 4F.1). Thus we shall need:

(4D.4) For any matrices $\tilde{\eta}, \tilde{\beta}$ with $\varphi(\tilde{\eta})=\eta$ and $\varphi(\tilde{\beta})=\beta$, one can construct the immersed spheres $G_{j}$ such that $E_{*} \cdot G_{*}=\tilde{\eta}, F_{*} \cdot G_{*}=\tilde{\beta}$.

Proof. It suffices to show that one can multiply the group element $\tilde{g}$ in the discussion preceding (4D.3) by $s^{ \pm 1}$, and that can clearly be done by letting the homotopy $f_{t}$ in the proof of (4D.1) run once extra around in the $S^{1}$-direction (positively or negatively) before "settling down" at 0 or $\frac{1}{2}$.

4E. Computing the self-intersections of $G_{*}$. Let $G_{*}=G_{1} \cup \cdots \cup G_{r}$. In this section we prove:

(4E.1) The self-intersection matrix $\tilde{x}$ of $G_{*}$ satisfies the equation

$$
(1-s) \tilde{x}=\tilde{\eta}^{s *} \tilde{\beta}-s \tilde{\theta}+(-1)^{k} \tilde{\theta}^{s *}
$$

for some $\tilde{\theta} \in M_{r}(\mathbf{Z} \pi)$, compare (2D.10).

Proof. We must put $G_{*}$ in general position before computing. This can be done by pushing along the $S^{1}$-direction alone. The various disks used to cap off the holes in the spheres with holes in (4D.2) are all disjoint. Thus, any double point for $G_{*}$ occurs above some double point path $D$ for $\Gamma_{*}$, and we may view $\tilde{x}$ as a sum of contributions from each $D$. Similarly, $\tilde{\eta}^{s *} \tilde{\beta}$ is, in a natural way, a sum of contributions from each double point path (just like $\eta^{*} \beta$ in (4B.3)). We shall construct $\tilde{\theta}$ as a similarly indexed sum. Thus the equation in (4E.1) will be proved by proving it for each double point path $D$ separately. 


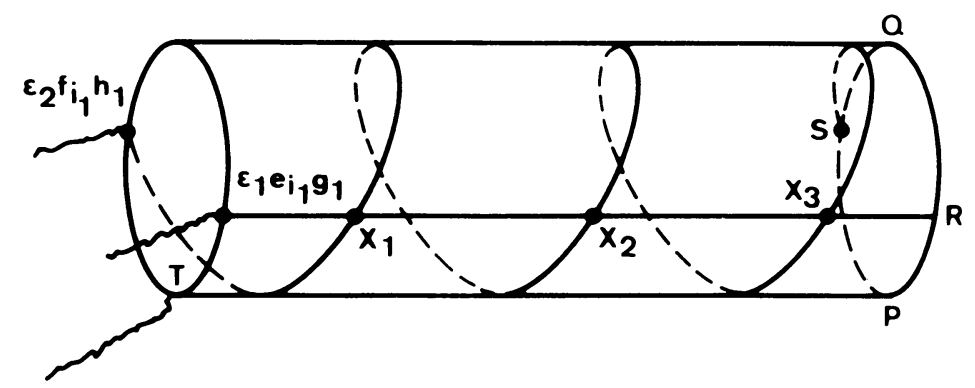

FIGURE 2

The inverse image $p^{-1}(D)$ of the double point path is a cylinder, and the trivialization can be chosen so that one of the double point paths goes straight across as in Figure 2. The other double point path will wind around somehow, some number of times, say $a$. After a regular homotopy fixing endpoints we may assume that the winding occurs with even speed as in the picture (where $a=+3$ ). We get double points $X_{1}, X_{2}, \ldots, X_{|a|}$ because of this winding. Irrespective of the type of $D$, we have a fixed situation at the left end of the picture: We have liftings of $S_{i_{1}}^{k} \times p t$ and $p t \times S_{i_{1}}^{k}$ with fundamental group element $g_{1}$ and $h_{1}$ in $\rho$, and with signs $\varepsilon_{1}, \varepsilon_{2}$. The associated intersection of $E_{i_{1}}$ and $F_{i_{1}}$ with $G_{j}$ have intersection "numbers" $\varepsilon_{1} \tilde{g}_{1}$ and $\varepsilon_{2} \tilde{h}_{1}$ as in the discussion preceding (4D.3). The wiggly line at $T$ symbolizes the path $\tilde{\sigma}_{i_{1}}$ from $S_{i_{1}}^{k} \times S_{i_{1}}^{k} \times S^{1}$ to the basepoint. Also, the two wiggly lines in the left-hand side represent paths from boundary components $S_{i_{1}}^{k} \times p t \times 0$ and $p t \times S_{i_{1}}^{k}$ $\times \frac{1}{2}$ through the "sphere with holes" $\hat{\Gamma}_{j}$ to its basepoint and along $\hat{\gamma}_{j}$ to the basepoint of $M$. Thus the loop from the basepoint to $T$, directly up to $S_{i_{1}}^{k} \times p t \times 0$ along $S^{1}$, and back to the basepoint through $\hat{\Gamma}_{j}$ and $\hat{\gamma}_{j}$, is $\tilde{g}_{1}$. Similarly, $\tilde{h}_{1}$ is represented by the path to $T$, directly up to $p t \times S_{i}^{k} \times \frac{1}{2}$ along the negative $S^{1}$-direction, and back to the basepoint through $\hat{\Gamma}_{j}$ and $\hat{\gamma}_{j}$.

Each double point $X_{i}$ comes with a sign and a group element in $\pi$. All $X_{i}$ obviously have the same sign, and consideration of a standard model of the situation reveals that it is given:

(4E.2) $\operatorname{sgn}\left(X_{i}\right)=\operatorname{sgn}(a) \cdot w\left(g_{1}\right) \cdot w_{B}\left(g_{1}\right) \cdot \varepsilon_{1} \varepsilon_{2} \quad\left(=\operatorname{sgn}(a) \cdot w_{E}\left(g_{1}\right) \cdot \varepsilon_{1} \varepsilon_{2}\right)$.

Throughout the rest of the proof we assume that $a \geqslant 0$, leaving the other case as a pleasant exercise. From the picture we see that:

(4E.3) The group elements associated to $X_{1}, X_{2}, \ldots, X_{a}$ are $\tilde{g}_{1}^{-1} \tilde{h}_{1}$, $\tilde{g}_{1}^{-1} s \tilde{h}_{1}, \ldots, \tilde{g}_{1}^{-1} s^{a-1} \tilde{h}_{1}$.

To get the information we need about $a$, we must distinguish between double point paths of type I and II, but we must also take into account whether the $S^{1}$-bundle is orientable or not along the path. In the first case we speak of type I.A or II.A, in the latter of type I.B or II.B. We claim that:

(4E.4) The number $a$ satisfies the equation

$$
\begin{aligned}
\tilde{g}_{1}^{-1} s^{a-1} \tilde{h}_{1} & =\tilde{g}_{2}^{-1} t \tilde{h}_{2}, & & D \text { of type I.A, } \\
& =\tilde{g}_{2}^{-1} \tilde{h}_{2}, & & D \text { of type I.B }, \\
& =\tilde{h}_{2}^{-1} \tilde{g}_{2}, & & D \text { of type II.A, } \\
& =\tilde{h}_{2}^{-1} s \tilde{g}_{2}, & & D \text { of type II.B. }
\end{aligned}
$$


Proof. Consider first case I.A. In Figure 2 one writes $\varepsilon_{3} e_{i_{2}} \tilde{g}_{2}$ at $R, \varepsilon_{4} f_{i_{2}} \tilde{h}_{2}$ at $S$. The path to the basepoint at the right-hand end is at $P$. Thus, we can compute the group element of the intersection point $X_{a}$ to be $\tilde{g}_{2}^{-1} t \tilde{h}_{2}$.

In case I.B one still writes $\varepsilon_{3} e_{i_{2}} \tilde{g}_{2}$ at $R$ and $\varepsilon_{4} f_{i_{2}} \tilde{h}_{2}$ at $S$. However, the orientation of $S^{1}$ has now been changed so the path to the basepoint now starts at $Q$ (recall that boundary components covering some $S_{i_{2}}^{k} \times p t$ occur only at level 0 in the $S^{1}$-factor, and the path to the basepoint starts at level $\frac{3}{4}$ ). The group element of $X_{a}$ is then computed to be $\tilde{g}_{2}^{-1} \tilde{h}_{2}$.

In cases II.A and B one has to write $\varepsilon_{3} f_{i_{2}} \tilde{h}_{2}$ at $R$ and $\varepsilon_{4} e_{i_{2}} \tilde{g}_{2}$ at $S$. For II.A one has the path to the basepoint starting at $Q$ and the group element of $X_{a}$ becomes $\tilde{h}_{2}^{-1} \tilde{g}_{2}$. And for II.B the basepoint path starts at $P$, and one gets $\tilde{h}_{2}^{-1} s \tilde{g}_{2}$.

REMARK. When we compute the self-intersections of $G_{*}$, we use the convention that the $e$-sheet comes before the $f$-sheet. However, in the above proof we must, of course, use the same sheet as the first one in both computations of the group element of $X_{a}$. Therefore, in cases II.A and B in the proof above, at the right-hand end of the cylinder, the $f$-sheet comes first.

We can use (4E.4) and (3B.1) to compute $\varepsilon_{1} \varepsilon_{2} \tilde{g}_{1}^{s *} s^{a} \tilde{h}_{1}$ in terms of $\varepsilon_{3} \varepsilon_{4} \tilde{g}_{2}^{s *} \tilde{h}_{2}$. The results are nice,

$$
\begin{aligned}
\varepsilon_{1} \varepsilon_{2} \tilde{g}_{1}^{s *}{ }^{a} \tilde{h}_{1} & =-\varepsilon_{3} \varepsilon_{4} \tilde{g}_{2}^{s *} \tilde{h}_{2}, \quad D \text { of type I, } \\
& =-(-1)^{k} \varepsilon_{3} \varepsilon_{4} s \tilde{h}_{2}^{s *} \tilde{g}_{2}, \quad D \text { of type II, } \omega\left(h_{2}\right)=1, \\
& =-(-1)^{k} \varepsilon_{3} \varepsilon_{4} t \tilde{h}_{2}^{s *} \tilde{g}_{2}, \quad D \text { of type II, } \omega\left(h_{2}\right)=-1,
\end{aligned}
$$

but the proof is rather tedious, since we have to divide it into 12 different cases. In fact, each of the types I.A and B divides into two cases according to $\omega\left(g_{1}\right)$. (Note that $\omega\left(g_{1}\right)=\omega\left(g_{2}\right)$ in type I.A, while $\omega\left(g_{1}\right)=-\omega\left(g_{2}\right)$ in type I.B.) And each of the types II.A and B divides into 4 cases depending on $\omega\left(g_{1}\right)$ and $\omega\left(h_{1}\right)$. (Note that $\omega\left(h_{2}\right)=\omega\left(g_{1}\right)$ and $\omega\left(g_{2}\right)=\omega\left(h_{1}\right)$ in type II.A, while $\omega\left(h_{2}\right)=-\omega\left(g_{1}\right)$ and $\omega\left(g_{2}\right)$ $=-\omega\left(h_{1}\right)$ in type II.B.) As an illustration, we do the case II.B, $\omega\left(g_{1}\right)=1, \omega\left(h_{1}\right)=$ -1 .

In that case, $\omega\left(g_{2}\right)=1$ and $\omega\left(h_{2}\right)=-1$ so

$$
\begin{aligned}
\varepsilon_{1} \varepsilon_{2} \tilde{g}_{1}^{s *} s^{a} \tilde{h}_{1} & =\varepsilon_{1} \varepsilon_{2} w_{E}\left(\tilde{g}_{1}\right) \tilde{g}_{1}^{-1} s^{a} \tilde{h}_{1} \\
& =\varepsilon_{1} \varepsilon_{2} w_{B}\left(g_{1}\right) s \tilde{g}_{1}^{-1} s^{a-1} \tilde{h}_{1} \\
& =-(-1)^{k} \varepsilon_{3} \varepsilon_{4} w_{B}\left(h_{2}\right) s \tilde{h}_{2}^{-1} s \tilde{g}_{2} \\
& =(-1)^{k} \varepsilon_{3} \varepsilon_{4} w_{E}\left(\tilde{h}_{2}\right) s \tilde{h}_{2}^{-1} s \tilde{g}_{2} \\
& =(-1)^{k} \varepsilon_{3} \varepsilon_{4} s \tilde{h}_{2}^{*} s \tilde{g}_{2} \\
& =-(-1)^{k} \varepsilon_{3} \varepsilon_{4} \tilde{h}_{2}^{s *} s \tilde{g}_{2} \\
& =-(-1)^{k} \varepsilon_{3} \varepsilon_{4} t \tilde{h}_{2}^{s *} \tilde{g}_{2} .
\end{aligned}
$$

The remaining 11 cases are treated similarly.

On the other hand, from (4E.2,3) one can immediately compute $(1-s) \tilde{x}_{D}$. One gets

$$
(1-s) \tilde{x}_{D}=\varepsilon_{1} \varepsilon_{2} e_{j k}\left(\tilde{g}_{1}^{s *}\left(1-s^{a}\right) \tilde{h}_{1}\right)
$$


(recall that $e_{j k}(y)$ is the $r \times r$ matrix with $y$ in position $(j, k)$, zeros elsewhere).

And one can compute $\tilde{\eta}^{s * \tilde{\beta}}$ to be

$$
\begin{aligned}
& \left(\tilde{\eta}^{s *} \tilde{\beta}\right)_{D}=e_{j k}\left(\varepsilon_{1} \varepsilon_{2} \tilde{g}_{1}^{s *} \tilde{h}_{1}\right)+e_{j k}\left(\varepsilon_{3} \varepsilon_{4} \tilde{g}_{2}^{s *} \tilde{h}_{2}\right), \quad D \text { of type I, } \\
& =e_{j k}\left(\varepsilon_{1} \varepsilon_{2} \tilde{g}_{1}^{s *} \tilde{h}_{1}\right)+e_{j k}\left(\varepsilon_{3} \varepsilon_{4} \tilde{g}_{2}^{s *} \tilde{h}_{2}\right), \quad D \text { of type II. }
\end{aligned}
$$

A little bit of bookkeeping based on (4E.7-9) reveals that $\tilde{\eta}^{s *} \tilde{\beta}-(1-s) \tilde{x}=s \tilde{\theta}-$ $(-1)^{k} \tilde{\theta}^{s *}$ when one takes $\tilde{\theta}$ to be given by

$$
\begin{aligned}
\tilde{\theta}_{D} & =0, & & D \text { of type I, } \\
& =\varepsilon_{3} \varepsilon_{4} t e_{k j}\left(\tilde{g}_{2}^{s *} \tilde{h}_{2}\right), & & D \text { of type II. }
\end{aligned}
$$

4F. Finishing the proof. The elements $E_{*}, F_{*}, G_{*}$ have a self-intersection form which is precisely the matrix $W_{3}$ of Proposition 2D.11 (see (4C.3), (4D.4) and (4E.1)). We might finish off the proof by purely algebraic considerations using the fact that the form $W_{3}$ is nonsingular on a direct summand of rank $2 r$, and the fact that $K_{k}\left(M^{\prime}\right)$ is also of rank $2 r$.

We do, however, prefer to have a completely geometric description of the surgery obstruction and only then refer to Lemma 2D.6 to show that it coincides with the algebraically defined obstruction of $\S 2 \mathrm{D}$. This strategy is based on

LEMMA 4F.1. If

$$
\left(\begin{array}{cc}
\tilde{a} & \tilde{\eta} \\
\tilde{z} & \tilde{\beta}
\end{array}\right) \in G l_{2 r}(\mathbf{Z} \pi) \text { and has } \varphi\left(\begin{array}{c}
\tilde{\eta} \\
\tilde{\beta}
\end{array}\right)=\left(\begin{array}{l}
\eta \\
\beta
\end{array}\right),
$$

then $K_{k+1}\left(M^{\prime}\right)$ has a $\mathbf{Z} \pi$ basis consisting of the elements $H_{j}=\sum_{i} E_{i} t \tilde{z}_{i j}^{s}+\sum_{i}(-1)^{k} F_{i} \tilde{a}_{i j}^{s}$, $j=1,2, \ldots, r$, and the elements $G_{j}, j=1,2, \ldots, r$.

Before proving the lemma let us note that the self-intersection form w.r.t. this basis gets the matrix $W_{2}$ of Lemma 2D.6 so that we do indeed finish the proof.

In the proof of the lemma we assume that $t$ has finite order $n$ (the alternative case is slightly easier). Also, recall the decomposition $M^{\prime}=M_{0} \cup V^{\prime}$ of $\S 4$.C. We shall use the 5-lemma and the diagram:

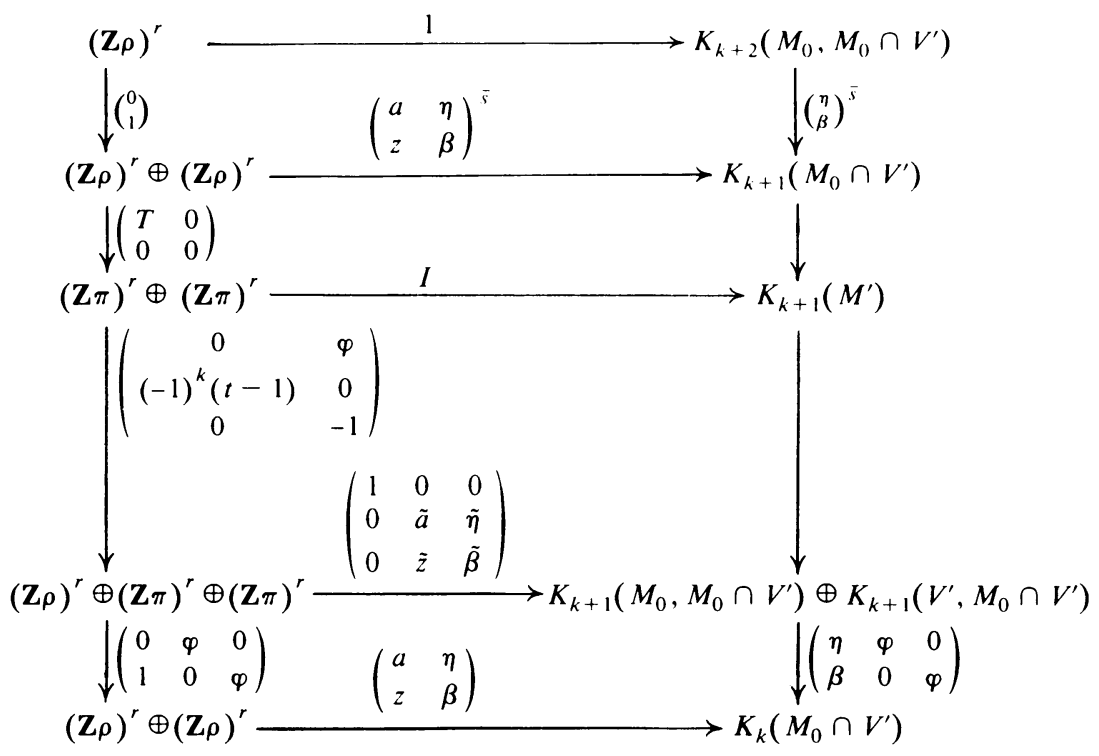


In the left-hand column we have $T=1+t+\cdots+t^{n-1}$, and the column is exact. The right-hand column is a Mayer-Vietoris sequence, hence alsc exact.

The Gysin sequence for $\left(M_{0}, M_{0} \cap V^{\prime}\right) \rightarrow\left(N_{0}, \partial U\right)$ gives isomorphisms $(\mathbf{Z} \rho)^{r}=$ $K_{k+1}\left(N_{0}, \partial U\right) \rightarrow K_{k+2}\left(M_{0}, M_{0} \cap V^{\prime}\right)$ and $K_{k+1}\left(M_{0}, M_{0} \cap V^{\prime}\right) \rightarrow K_{k+1}\left(N_{0}, \partial U\right)=$ $(\mathbf{Z} \rho)^{r}$. Both of these appear as equalities in the diagram. Also $M_{0} \cap V^{\prime}=\cup_{i} S_{i}^{k} \times$ $S_{i}^{k} \times S^{1}$ so $K_{k+1}\left(M_{0} \cap V^{\prime}\right)$ and $K_{k}\left(M_{0} \cap V^{\prime}\right)$ are free $\mathbf{Z} \rho$-modules with bases $S_{i}^{k} \times p_{i} \times S^{1}, q_{i} \times S_{i}^{k} \times S^{1}(i=1,2, \ldots, r)$ and $S_{i}^{k} \times p_{i} \times 0, q_{i} \times S_{i}^{k} \times 0$, respectively. Finally, $K_{k+1}\left(V^{\prime}, M_{0} \cap V^{\prime}\right)=K_{k+1}\left(V^{\prime}, \partial V^{\prime}\right)$ is a free $\mathbf{Z} \pi$-module with basis $D_{i}^{k+1} \times p_{i} \times 0$ and $q_{i} \times D_{i}^{k+1} \times \frac{1}{2}(i=1,2, \ldots, r)$.

In the left-hand side all modules are given their obvious $\mathbf{Z} \rho$ - or $\mathbf{Z} \pi$-bases. We can then specify the maps in terms of matrices. All the horizontal arrows given in terms of the matrix

$$
\left(\begin{array}{ll}
\tilde{a} & \tilde{\eta} \\
\tilde{z} & \tilde{\beta}
\end{array}\right)
$$

are isomorphisms (of course, $a=\varphi(\tilde{a}), z=\varphi(\tilde{z})$ ), and the map $I$ sends the standard basis to the elements $H_{1}, \ldots, H_{r}, G_{1}, \ldots, G_{r}$. Thus, the proof is complete if we verify that the diagram commutes.

The map from $K_{k+2}\left(M_{0}, M_{0} \cap V^{\prime}\right)$ to $K_{k+1}\left(M_{0} \cap V^{\prime}\right)$ is given by $\left(\begin{array}{l}\eta \\ \beta\end{array}\right)^{\bar{s}}$ rather than $\left(\begin{array}{l}\eta \\ \beta\end{array}\right)$ because in the diagram

$$
\begin{array}{ccc}
K_{k+1}\left(N_{0}, \partial U\right) & = & K_{k+2}\left(M_{0}, \partial V^{\prime}\right) \\
\downarrow \partial=\left(\begin{array}{l}
\eta \\
\beta
\end{array}\right) & & \downarrow \partial \\
K_{k}(\partial U) & & K_{k+1}\left(\partial U \times S^{1}\right)
\end{array}
$$

the operation $-\times S^{1}$ commutes with the action of $\rho$ only up to the sign given by $\omega$. Once this is realized, it is clear that the top square commutes.

In the next square $S_{i}^{k} \times p_{i} \times S^{1}$ and $q_{i} \times S_{i}^{k} \times S^{1}$ map to $(-1)^{k} F_{i} T$ and $E_{i} T$, respectively. A direct computation then shows the square commutative.

Next, the elements $E_{i}, F_{i}, G_{i}$ of $K_{k+1}\left(M^{\prime}\right)$ are seen to map to

$$
(-1)^{k}\left(q_{i} \times D_{i}^{k+1} \times \frac{1}{2}\right)(1-s),\left(D_{i}^{k+1} \times p_{i} \times 0\right)(t-1)
$$

and

$$
g_{i}-\sum_{j}\left(D_{j}^{k+1} \times p_{j} \times 0\right) \tilde{\eta}_{j i}-\sum_{j}\left(g_{j} \times D_{j}^{k+1} \times \frac{1}{2}\right) \tilde{\beta}_{j i}
$$

respectively. Here $g_{i}, i=1,2, \ldots, r$, is the $\mathbf{Z} \rho$ basis for $K_{k}$. Hence, the third square commutes.

Finally, the last map on the right-hand side obviously is as indicated so the bottom square commutes.

\section{REFERENCES}

1. H. J. Munkholm and E. K. Pedersen, Whitehead transfers for $S^{\prime}$-bundles, an algebraic description, Comment. Math. Helv. 56 (1981), 404-430.

2. E. K. Pedersen, Universal geometric examples for transfer maps in algebraic $K$ - and L-theory, J. Pure Appl. Algebra 22 (1981), 179-192. 